\title{
X-RAY PROPERTIES OF PROTOSTARS IN THE ORION NEBULA
}

\author{
L. Prisinzano, ${ }^{1}$ G. Micela, ${ }^{1}$ E. Flaccomio, ${ }^{1}$ J. R. Stauffer, ${ }^{2}$ T. Megeath, ${ }^{3}$ L. Rebull ${ }^{2}$ \\ M. Robberto, ${ }^{4}$ K. Smith, ${ }^{4}$ E. D. Feigelson, ${ }^{5}$ N. Grosso, ${ }^{6}$ and S. Wolk ${ }^{7}$ \\ Received 2007 November 5; accepted 2007 December 17
}

\begin{abstract}
The origin and evolution of the X-ray emission in very young stellar objects (YSOs) are not yet well understood because it is very hard to observe YSOs in the protostellar phase. Using COUP data, we study the X-ray properties of stars in the ONC in different evolutionary classes: luminosities, hydrogen column densities $N_{\mathrm{H}}$, effective plasma temperatures, and time variability are compared to understand if the interaction between the circumstellar material and the central object can influence the X-ray emission. We have assembled the deepest and most complete photometric catalog of objects in the ONC region from the UV to $8 \mu \mathrm{m}$ using data from the HST Treasury Program; deep and almost simultaneous $U B V I$ and $J H K$ images taken, respectively, with WFI at ESO $2.2 \mathrm{~m}$ and ISPI at CTIO $4 \mathrm{~m}$ telescopes; and Spitzer IRAC imaging. We select high-probability candidate Class 0-I protostars, a sample of "bona fide" Class II stars, and a set of Class III stars with IR emission consistent with normal photospheres. Our principal result is that Class 0-Ia objects are significantly less luminous in X-rays, in both the total and hard bands, than the more evolved Class II stars with mass larger than $0.5 M_{\odot}$; the latter show X-ray luminosities similar to those of Class $0-\mathrm{Ib}$ stars. This result supports the hypothesis that the onset of X-ray emission occurs at a very early stage of star formation. Spectral properties of Class 0 -I stars are similar to those of the more evolved Class II and III objects, except for a larger absorption likely due to gas in the envelope or disk of the protostellar objects. Our data suggest that the three different classes have similar X-ray temporal variability.
\end{abstract}

Subject headings: open clusters and associations: general — open clusters and associations:

individual (Orion Nebula Cluster) — stars: activity — stars: pre-main-sequence -

X-rays: stars

Online material: machine-readable tables

\section{INTRODUCTION}

Young stellar objects (YSOs) exhibit an X-ray luminosity significantly higher than similar stars in the main-sequence phase. However, it is still unclear at what stage of their formation process YSOs begin to emit X-rays and how this X-ray emission evolves with time (Favata \& Micela 2003; Feigelson et al. 2007).

Evidence has accumulated over the past few years that among the YSOs, the X-ray luminosity function (XLF) of classical T Tauri stars (CTTSs) is different from that of weak T Tauri stars (WTTSs). Such a difference has been investigated by some because the accretion and outflow processes, characteristic of the CTTS phase, involve the interaction of ionized material with the star and disk magnetic field, and these media play a fundamental role in the coronal X-ray emission of young stars. Using the Chandra Orion Ultradeep Project (COUP) data (Getman et al. 2005a), Preibisch et al. (2005) find that the accreting stars, as discriminated using the Ca II $\lambda 8542$ IR triplet line, are less X-ray luminous than nonaccretors, while no differences are found for stars with $(K-L)$ IR excesses, indicative of the presence of a (possibly passive) disk, with respect to stars without IR excesses. These results are in agreement with those found in the previous work in the Orion

\footnotetext{
1 INAF, Osservatorio Astronomico di Palermo, I-90134 Palermo, Italy; loredana@astropa.inaf.it.

2 Spitzer Science Center, California Institute of Technology, Pasadena, CA 91125.

3 Department of Physics, University of Toledo, Toledo, OH 43606.

4 Space Telescope Science Institute, Baltimore, MD 21218.

Department of Astronomy and Astrophysics, Pennsylvania State University, University Park, PA 16802.

6 Observatoire Astronomique de Strasbourg, Université Louis-Pasteur, CNRS, INSU, 67000 Strasbourg, France.

7 Harvard Smithsonian Center for Astrophysics, Cambridge, MA 02138.
}

Nebula Cluster (ONC) by Flaccomio et al. (2003a, 2003c), who found a strong difference in the X-ray luminosity of accreting and nonaccreting stars.

In order to understand the evolution of the X-ray emission during the star formation process, Preibisch \& Feigelson (2005) looked for a possible relation between X-ray emission and stellar age in the ONC. They found, in the age range $\sim 0.1-10 \mathrm{Myr}$, a slow decay in the X-ray luminosity, $L_{\mathrm{X}}$. Because, as the star contracts, the bolometric luminosity, $L_{\mathrm{bol}}$, decreases faster than $L_{\mathrm{X}}$, on average $L_{\mathrm{X}} / L_{\mathrm{bol}}$ and the $\mathrm{X}$-ray surface flux, $F_{\mathrm{X}}$, increase during this age range.

In this work, we want to investigate the onset and the evolution of X-ray emission during the early phase of the star formation by statistically comparing the X-ray properties of the stars in different evolutionary phases rather than considering individual stellar ages, usually derived from theoretical isochrones. We hope in this way to avoid some of the problems introduced when using inferred ages (due to their often large random and systematic uncertainties; see, e.g., Hillenbrand et al. 2007).

YSOs in their early phases are characterized by IR excesses due to the presence of a circumstellar envelope and/or a disk that contains warm dust. The first IR classification in Class I, II, and III sources, which is based on the IR spectral index, was proposed by Lada (1987). The discovery in the submillimeter of cold objects, younger than the IR sources, leads to the introduction of the Class 0 sources (Andre et al. 1993). Based on their mid-IR properties, YSOs are usually classified as follows (e.g., Allen et al. 2004, 2007; Megeath et al. 2004; Lada et al. 2006): Class 0 objects are protostars surrounded by the initial infalling envelope, typically detected in the submillimeter and far-IR; Class I objects are evolved protostars surrounded by an envelope and a circumstellar disk; Class II stars are objects with a circumstellar disk but lacking significant evidence for a dense envelope; and finally, 
TABLE 1

Log of the New Optical-IR Observations Used in This Work

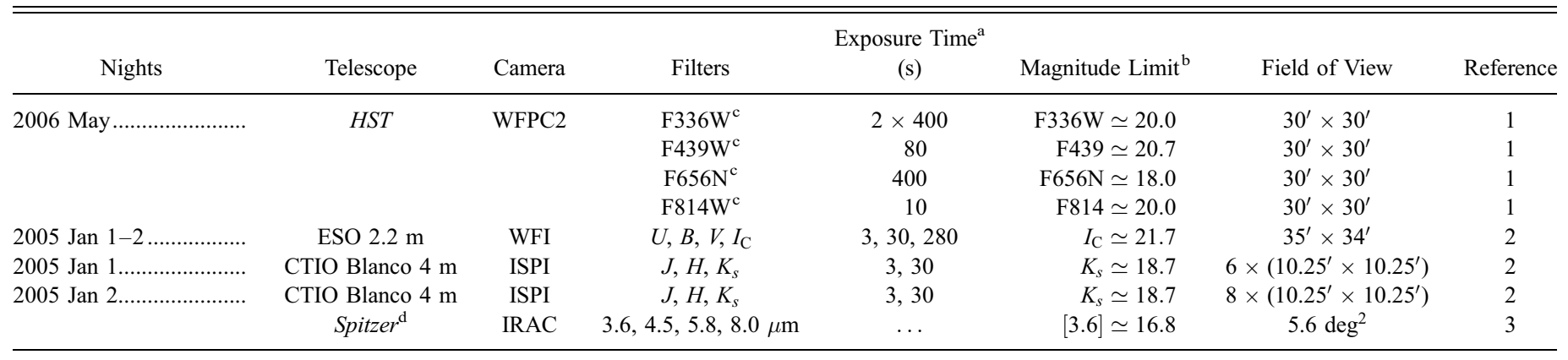

a Exposure time per filter.

b Magnitude limit computed for stars with photometric errors smaller than 0.1 .

c WFPC2 filters F336W, F439W, F656N, and F814W correspond to the standard filters $U, B$, H $\alpha$, and $I$, respectively.

d Data part of the Spitzer GTO Programs 43 and 50 taken in high dynamic range mode using 0.6 and $12 \mathrm{~s}$ at each position.

REFERENCES.-(1) Robberto et al. 2005b; (2) M. Robberto et al. 2008, in preparation; (3) Megeath et al. 2005.

Class III stars are objects that have lost their initial envelope and disk and show optical and near-IR (NIR) colors consistent with normal photospheres.

By providing very sensitive 3.6-70 $\mu \mathrm{m}$ data with good angular resolution, Spitzer observations have provided a much better census of the stellar populations in a number of star-forming regions. However, Spitzer data have also complicated the YSO classification system, allowing detection of some Class 0 objects at wavelengths as blue as $3.6 \mu \mathrm{m}$.

Our intent is to compare the X-ray properties of protostars (where to date there are relatively few studies) with the X-ray properties of the more evolved Class II and III stars, for which the X-ray emission is well studied. Such a comparison is fundamental in order to understand the onset of X-ray emission in YSOs and its evolution in the early phases of star formation.

Previous studies of X-ray emission from Class $0-\mathrm{I}$ objects include Ozawa et al. (2005), who studied core F of the $\rho$ Ophiuchi cloud and found a high X-ray detection rate (64\%) of Class I sources. They suggest that an evolutionary effect in temperature and extinction is present from Class I to Class III stars, the latter having lower coronal temperatures and extinction.

A similar result has also been found by Giardino et al. (2007). Getman et al. (2007) studied the IC 1396N cometary globule where they found six X-ray-emitting protostars, including the Class 0-I IRAS $21391+5802$, which they claim to be one of the youngest objects ever detected in X-rays. The comparison of X-ray emission from different evolutionary classes is, however, hampered by the difficulty of detecting X-rays from Class 0 protostars. For example, no X-ray emission has been found from Class 0 stars in the Serpens, one of the most sensitive surveys (Giardino et al. 2007), even if important detections have been found in other surveys (e.g., Tsuboi et al. 2001; Hamaguchi et al. 2005; Tsujimoto et al. 2005; Getman et al. 2007).

The ONC is a prime target for this kind of investigation. It is the nearest concentrated site of star formation with a very large number of known members covering all evolutionary stages. It is the best studied region at all wavelengths, in particular, in the X-ray band. Many interesting results have been obtained with the extraordinarily long Chandra observation performed by the COUP. To understand the evolution of X-ray activity from the very first stages of the star formation process, it is necessary to distinguish Class 0-I objects from Class II stars. Such a classification, based on the shape of the spectral energy distribution (SED), is not available in the literature and requires complete and deep NIR and mid-IR data.
We present here the results of the X-ray study of YSOs in the ONC classified using the Spitzer IRAC catalog (Megeath et al. 2005), ground-based $J H K$ data (M. Robberto et al. 2008, in preparation), and the deep photometric catalog obtained within the Hubble Space Telescope (HST) Treasury Program (Robberto et al. $2005 b$ ). Using the COUP data, we have studied the X-ray properties of YSOs from their initial protostellar phase (Class 0-I objects) to the more evolved pre-main-sequence (PMS) stage of no longer accreting and disk-free stars (Class III objects). We have classified the ONC members using conservative criteria in order to minimize the contamination of the resulting samples. Because of the large area coverage and the richness of the ONC, we have obtained a homogeneous and statistically significant sample of X-ray-detected Class $0-\mathrm{I}$ objects. We are thus able, for the first time, to compute the XLF of Class $0-I$ stars and to compare it with the XLF of Class II and III stars.

\section{OBSERVATIONS}

We have assembled a deep photometric catalog of the ONC from the UV to $8 \mu \mathrm{m}$ obtained using new optical/NIR observations taken within the HST Treasury Program (Robberto et al. $2005 b$ ), using a variety of telescopes and detectors, and Spitzer IRAC (Werner et al. 2004; Fazio et al. 2004) imaging. The optical photometry was derived using images taken with the Wide Field Planetary Camera 2 (WFPC2) of HST (Robberto et al. 2005b) and with the Wide Field Imager (WFI) at the ESO $2.2 \mathrm{~m}$ Telescope (M. Robberto et al. 2008, in preparation). JHK photometry was obtained from images taken with the Infrared Side Port Imager (ISPI) camera at the Cerro Tololo Inter-American Observatory (CTIO) Blanco $4 \mathrm{~m}$ telescope (M. Robberto et al. 2008, in preparation). Spitzer IRAC photometry in the four standard filters centered on 3.6, 4.5, 5.8, and $8.0 \mu \mathrm{m}$ was obtained by Megeath et al. (2005). A detailed description of the optical-IR observations is given in Table 1. We note that the magnitude limits given in this table are typical values for each survey. However, due to the complex structure of the nebulosity in the ONC (and due to varying integration times for different parts of the region), the sensitivity is not uniform. For example, the limiting magnitude at $3.6 \mu \mathrm{m}$ is about 16 within most of the COUP field of view (FOV), while it is about 10 within a region of $1^{\prime}$ in radius around the brightest stars of the Trapezium cluster.

\subsection{X-Ray Data}

$\mathrm{X}$-ray data used in this work were obtained with the $\sim 838 \mathrm{ks}$ COUP observation of the ONC, taken with the ACIS camera that 
covers an FOV of $17^{\prime} \times 17^{\prime}$. Details of data reduction and the derived catalog of 1616 sources can be found in Getman et al. (2005a).

\subsection{Optical Data}

New optical photometric data taken with the WFPC2 camera of $H S T$ were used by Robberto et al. (2005b) to derive the optical photometric catalog used in this work. This catalog contains 1754 selected point sources with at least a detection in the F814 band.

The recent $H S T$ survey has been complemented with $U B V I$ images taken with the WFI camera of the ESO $2.2 \mathrm{~m}$ Telescope. Details on data reduction and photometric catalog derivation will be described in M. Robberto et al. (2008, in preparation). This catalog contains 2744 selected point sources with at least a detection in the $I$ band.

Additional optical data were retrieved from literature, including the catalogs of Hillenbrand (1997, hereafter H97), Rebull et al. (2000), and Herbst et al. (2002).

\subsection{JHK Data}

The $J H K$ photometric catalog has been obtained using images taken with the ISPI camera at the CTIO Blanco $4 \mathrm{~m}$ Telescope. Details on data reduction and photometry will be given in M. Robberto et al. (2008, in preparation). The survey includes a mosaic of 11 fields, each of $10.25^{\prime} \times 10.25^{\prime}$, centered around the Trapezium cluster. All fields were observed once using a fivepoint dithering pattern. From the source catalog we excluded saturated objects, that is, those with a peak flux in each band higher than 37,000 counts and entries with errors in magnitudes larger than 0.2. After this selection, we were left with 17,824 entries in the table.

Finally, we constructed a consolidated catalog of individual objects by merging entries with positions within $0.6^{\prime \prime}$, the radius at which spurious identifications start to dominate. For each object, we computed the mean coordinates and magnitudes. Our final $J H K$ photometric catalog contains 13,987 objects.

Additional $J H K$ data were retrieved from the Two Micron All Sky Survey (2MASS) catalog (Cutri et al. 2003). ${ }^{8}$

\subsection{Spitzer IRAC Data}

A description of the data, the reduction procedure, and the derived photometric catalog obtained from the Spitzer IRAC images is given in Megeath et al. (2005). The survey covers the Orion A and B molecular clouds for a total of $5.6 \mathrm{deg}^{2}$ with a sensitivity of $17.0,16.0,14.5$, and $13.5 \mathrm{mag}$ in the $3.6,4.5,5.8$, and $8.0 \mu \mathrm{m}$ bands, respectively. The uncertainties in the calibration are about $5 \%$, but for a few stars they are significantly worse due to the bright nebulosity in the 5.8 and $8.0 \mu \mathrm{m}$ bands. Within the region studied in this work, i.e., the COUP FOV, the catalog includes 1244 sources detected at both 3.6 and $4.5 \mu \mathrm{m}$ and 334 sources detected at both 5.8 and $8.0 \mu \mathrm{m}$. The Spitzer Orion Survey also included MIPS observations at $24 \mu \mathrm{m}$ that we do not use in the following analysis since they are saturated in the COUP region due to the high background from the $\mathrm{H}$ II region.

\subsection{Cross Identifications of Sources}

All the photometric catalogs obtained from the observations described in Table 1 were assembled in a single database. Common objects between two catalogs were found using the nearest neighbor method by first considering two sources as the same object if their positions agree to within a specified amount $\left(1^{\prime \prime}-3^{\prime \prime}\right)$

\footnotetext{
${ }^{8}$ See http://irsa.ipac.caltech.edu/applications/Gator/.
}

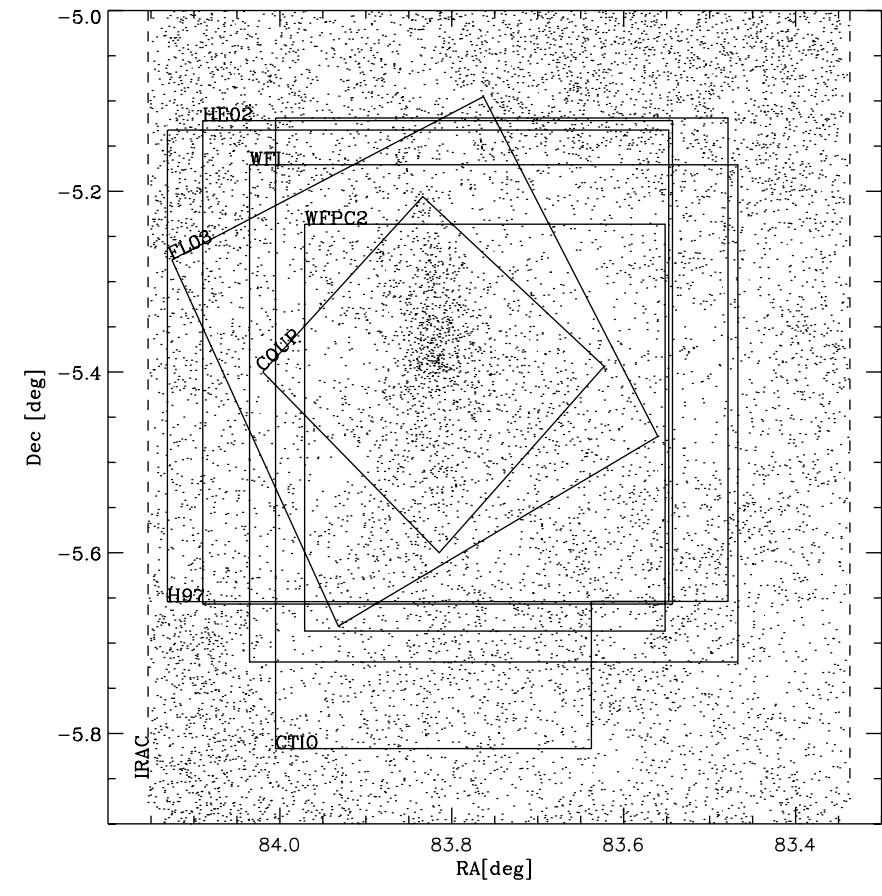

FIG. 1.-FOVs of the catalogs considered in this work overplotted on the spatial distribution of the stars detected with IRAC. Note that the FOVs of the IRAC (dashed line), Rebull et al. (2000), and 2MASS data (not indicated here) are larger than the field shown in this figure.

depending on the absolute astrometric accuracy of the catalogs. Matched sources were then used to compute the median and the standard deviation of the offsets. These median values were used to remove the systematic offsets between each pair of matched catalogs, and new matches were performed, for all pairs of catalogs, using new tolerances computed using the standard deviation of the offsets.

Using the same matching procedure, we have cross-correlated these data with the literature catalogs of H97, Rebull et al. (2000), 2MASS (Cutri et al. 2003), Herbst et al. (2002), and Muench et al. (2002) and the X-ray source list from the COUP Survey (Getman et al. 2005a).

Finally, we collected all data from all the cross-matched catalogs, including all objects detected in at least one catalog.

The FOVs covered by these surveys are overplotted in Figure 1 on the spatial distribution of the stars detected with IRAC around the ONC. Note that the FOVs of the IRAC, Rebull et al. (2000), and 2MASS data (not indicated) are larger than the field shown in the figure.

Since this work is focused on the X-ray properties of the young stars in the ONC, only stars within the COUP FOV are considered in the following analysis. In addition, photometric data with errors smaller than 0.2 mag are considered.

\section{YSO CLASSIFICATION}

In this section we describe how we select ONC stars belonging to the three different evolutionary classes $0-$ I, II, and III. Our approach is to select conservative, even if incomplete, samples in order to be able to compare the X-ray properties of the stars in different evolutionary stages using samples that are as "uncontaminated" as possible.

\subsection{Class 0-I Protostars}

According to Lada \& Wilking (1984) and Adams et al. (1987), the first stage of YSO evolution is the infall phase around a central 
protostar undergoing accretion, characterized by strong emission in the submillimeter and far-infrared and weak emission shortward of $24 \mu \mathrm{m}$. These objects, indicated as Class 0 , can have outflows and are defined as protostars with half or more of their mass in the envelope. More evolved protostars are the Class I objects with both a disk and envelope surrounding the central star. Both these classes of protostars are characterized by a steeply rising SED in the infrared range, although pure Class 0 objects are expected to be fainter at NIR and mid-IR bands (Allen et al. 2007).

Since our data extend only to $8 \mu \mathrm{m}$, we cannot distinguish between Class 0 and I stars, and we combine them into one group as Class 0-I protostars; we classify them using the following criterion: we computed $\log \left(\lambda F_{\lambda}\right)$ over the wavelengths $\left[\lambda_{1}, \ldots, \lambda_{5}\right]=$ $[2.2,3.6,4.5,5.8,8.0] \mu \mathrm{m}$, i.e., the $K$ band and the four IRAC bands with the Lada et al. (2006) flux conversion factors that are in agreement with the Reach et al. (2005) values; for each star we then computed the slopes for each pair of adjacent bands

$$
\frac{\log \left(\lambda_{i+1} F_{\lambda_{i+1}}\right)-\log \left(\lambda_{i} F_{\lambda_{i}}\right)}{\log \lambda_{i+1}-\log \lambda_{i}} \quad \text { for } i=1,4
$$

We consider Class 0-I objects those for which the slopes are all larger than 0, i.e., objects with rising SEDs. This criterion is more conservative than using the single slope (usually indicated as spectral index) computed from the linear fit of the $\log \left(\lambda F_{\lambda}\right)$ function over the whole wavelength range because it only selects monotonically increasing SEDs. Moreover, it is more robust than those based on only a color-color diagram (CCD) since it is based simultaneously on all the known magnitudes.

Our sample of Class $0-\mathrm{I}$ objects includes all the objects for which we have at least three of the five magnitudes and therefore at least two slopes. This allows us to include objects detected only in the $K, 3.6 \mu \mathrm{m}$, and $4.5 \mu \mathrm{m}$ bands but not at 5.8 and $8.0 \mu \mathrm{m}$; because of the bright nebular background and the lower sensitivity at $8 \mu \mathrm{m}$, many objects are not detected in these two bands (roughly $44 \%$ of the Class $0-$ I sample).

Using this criterion, we end up with 41 potential Class $0-\mathrm{I}$ objects in the COUP FOV (Getman et al. 2005a). Eighteen of them have a detection in at least one of the optical UBVI bands. Since protostars are expected to be heavily embedded and therefore highly reddened objects, we do not expect to detect them in the visible. Of these 18 peculiar objects, 5 (COUP sources 693, 747, $826,948,1011)$ are in the list of the X-ray-detected proplyds given in Kastner et al. (2005). From a visual inspection of HST Advanced Camera for Surveys (ACS) images, we conclude that all 18 of these objects have a counterpart in the HST optical bandpasses, while the other 23 objects are not detected at these bands. We eliminate these 18 objects from our list of candidate Class $0-I$ stars and consider the remaining 23 stars as Class 0-Ia objects to distinguish them from the additional sample of candidate Class 0 I objects described below. The SEDs of the 23 selected Class 0 Ia stars are plotted in Figure 2. The photometry is given in Table 2, where column (1) gives the sequential number in our catalog, column (2) is the COUP identification number, columns (3) and (4) are the J2000.0 coordinates, and columns (5)-(11) give the JHK and IRAC magnitudes. Of these 23 objects, $10(\sim 40 \%)$ have an $\mathrm{X}$-ray counterpart in the COUP data. One of these (COUP 702) is in the Getman et al. (2005b) list of nonflaring COUP X-ray sources without optical and NIR counterparts and was flagged by them as a probable extragalactic object (EG).

Figure 3 shows the CCDs obtained using the $K$ and IRAC magnitudes of all the stars in the COUP FOV.
The left panel includes more stars than the middle and right panels because it does not require detections at 5.8 and $8.0 \mu \mathrm{m}$. The limited sensitivity of the $8.0 \mu \mathrm{m}$ channel means that few objects with zero color (consistent with photospheric emission) appear in the right panel. The selected Class 0 -Ia objects with increasing SED, indicated in Figure 3 by squares, show [3.6] $[4.5]>0.7$ and $[5.8]-[8.0] \gtrsim 1.1$. The objects with [3.6] $[4.5]>0.7$ and $[5.8]-[8.0] \lesssim 1.1$ that are usually also classified as Class 0-I stars (Allen et al. 2004; Megeath et al. 2004) show a decreasing or flat SED at wavelengths longer than $4.5 \mu \mathrm{m}$. The SEDs of protostars in this spectral regime are complex as rotation (Kenyon et al. 1993) and outflow cavities (Whitney et al. 2003) reduce the extinction toward the poles of the protostellar envelopes allowing radiation to leak and scatter out. Consequently, protostars can also exhibit decreasing slopes, particularly between 4.5 and $8.0 \mu \mathrm{m}$, depending on their inclination (Whitney et al. 2003). Furthermore, highly reddened Class II objects can have increasing slopes; however, these slopes will generally flatten or decline for $\lambda \geq 4.5 \mu \mathrm{m}$ due to the flattening of the reddening law and the broad silicate feature at $9.7 \mu \mathrm{m}$ (Flaherty et al. 2007). Thus, although the IRAC data can enhance the identification of protostars by extending the SEDs to $8 \mu \mathrm{m}$, establishing a sample of protostars uncontaminated by reddened Class II objects is still difficult without photometry at wavelengths $\lambda>8 \mu \mathrm{m}$.

To take into account these cases, we also include the objects with a decreasing SED for $\lambda>4.5 \mu \mathrm{m}$ in our Class $0-$ I sample. There are 28 such objects in total. After rejecting the six objects with optical counterparts in the HST imaging, we are left with 22 objects, which we refer to hereafter as Class $0-$ Ib sources. The SEDs of the Class 0-Ib objects are shown in Figure 4, while the photometry is given in Table 3, analog to Table 2. Among these objects, $18(\sim 80 \%)$ are in the COUP list of X-ray detection, including COUP source 1565, which was classified as an extragalactic object by Getman et al. (2005b).

If we consider both Class $0-\mathrm{Ia}$ and $0-\mathrm{Ib}$ samples, our list of X-ray-detected Class 0 -I objects $(10+18)$ is one of the largest samples of Class $0-\mathrm{I}$ stars with evidence of X-ray emission (Getman et al. 2007; Giardino et al. 2007).

Our selected Class $0-\mathrm{I}$ objects fall in the region of the envelope models indicated in Allen et al. (2004) and Megeath et al. (2004). There are a small number of objects falling in the Class $0-$ I regions of the CCD that were not selected with our criterion because either they have an optical counterpart $(18+6$ in total) or their SED band-to-band slopes do not increase as required by our criterion. Whereas some of these rejected objects could be Class $0-\mathrm{I}$ stars, by adopting a conservative selection criterion, we have minimized the adverse effect of sample contamination for our comparison of X-ray properties.

A careful examination of the NIR and IRAC colors of our Class 0-I candidates (see Figs. 3 and 5) suggests to us that the Class 0-Ia candidates (on average) are inconsistent with their being heavily reddened Class II objects. In particular, the bottom right panel of Figure 5 shows that, using the Orion A reddening law of Flaherty, the Class 0-Ia candidates are too red in [4.5] [5.8] to be heavily reddened Class II objects, and in any case they would require reddenings of $A_{V} \geq 60$. The bottom left panel of Figure 5 also indicates that the Class 0 -Ia candidates, on average, would need very large reddenings $\left(A_{V} \sim 50\right)$ for them to be Class II sources. Such heavily embedded objects would likely be very young in any case, and we therefore believe that it is more likely that they are, indeed, Class 0 or Class I objects. By contrast, the Class $0-\mathrm{Ib}$ objects are located in all of the diagrams in Figures 3 and 5 in positions consistent with being moderately reddened $\left(A_{V} \sim 30\right.$ on average) Class II objects. We suspect that 

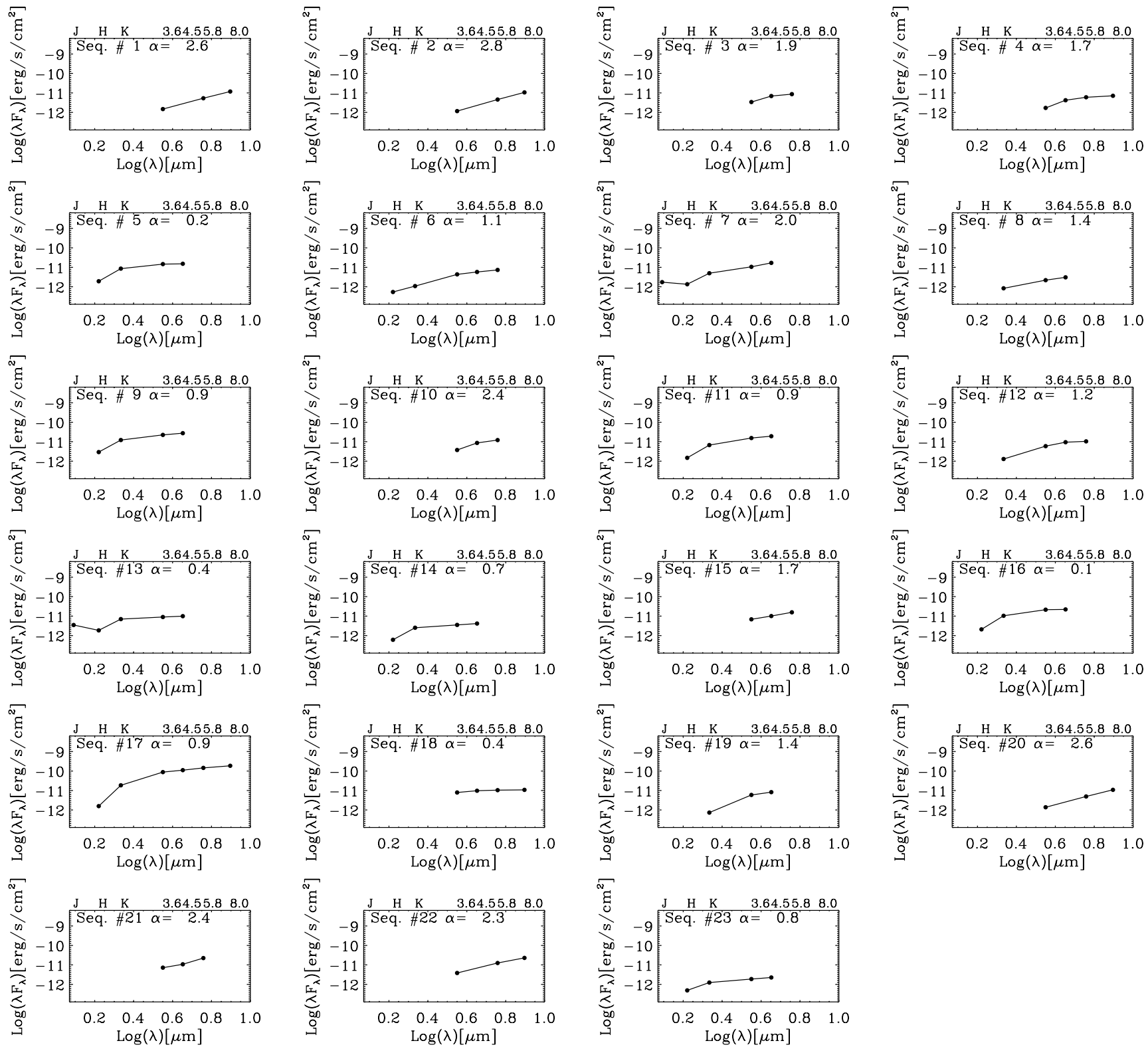
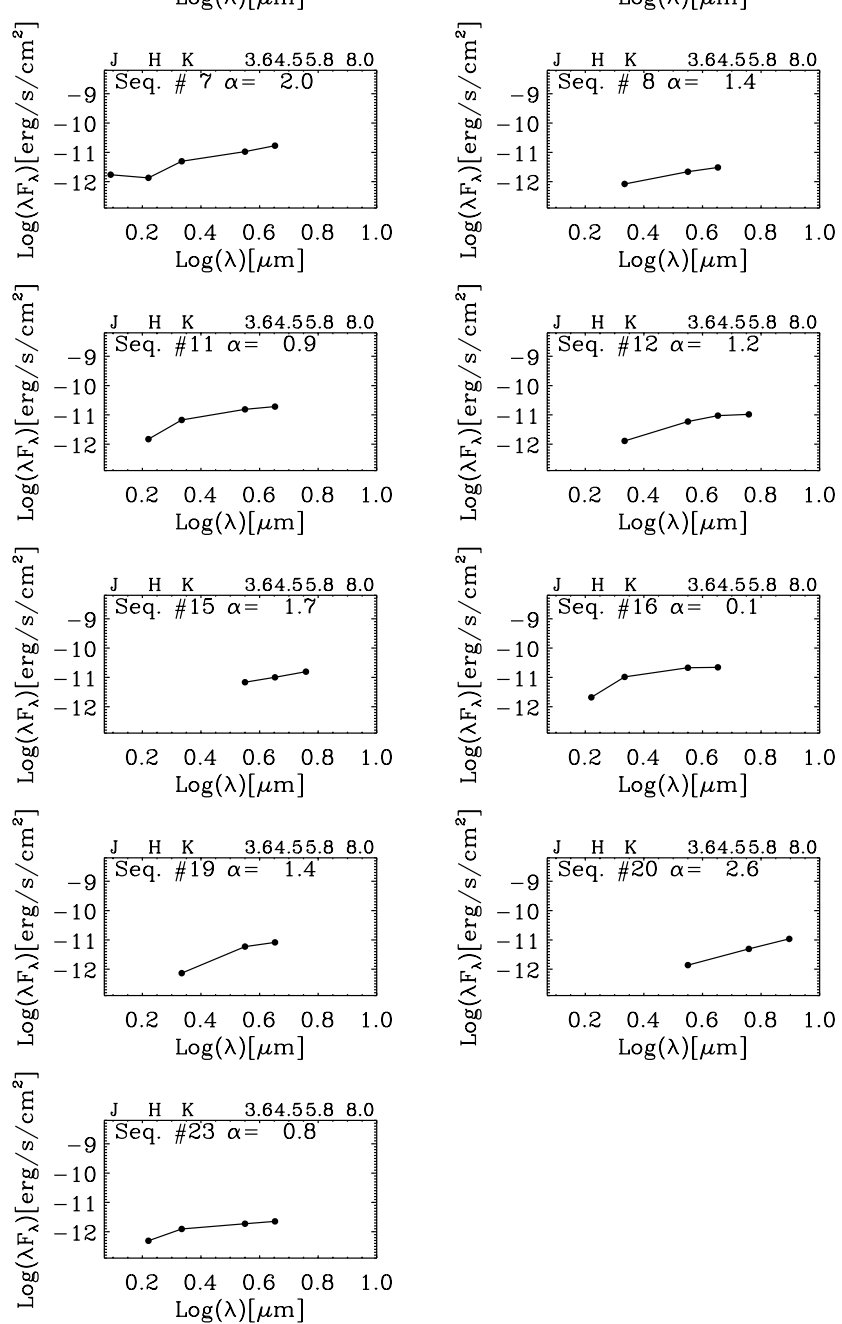

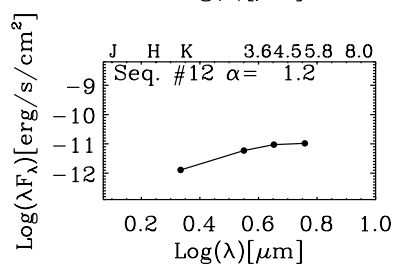

FIG. 2.-SED of the 23 Class 0 -Ia candidate objects with a rising SED from $K$ to $8.0 \mu \mathrm{m}$. The sequential number in our catalog and the spectral index computed using the available IRAC magnitudes are also indicated.

the Class $0-$ Ib objects are a mixture of true Class 0 or I objects and heavily reddened Class II objects; without more data we cannot clean the sample any further than we have.

Another possible worry is that our Class 0 -I candidate sample includes systems seen edge-on in or around the $\mathrm{H}$ II region. In these cases, relatively evolved Class II objects may display an SED peaking at mid-IR and far-IR wavelengths, as suggested by Robberto et al. (2002). However, since edge-on disks should be randomly distributed in the nebula, clustering in the dark regions of the selected objects (see Fig. $6 a$ ) strongly suggests that they are real protostars.

\subsection{Class II Stars}

Following Hartmann et al. (2005), Class II objects within the COUP FOV were selected using the conditions $0.2<[3.6]-$ $[4.5]<0.7$ and $0.6<[5.8]-[8.0]<1.1$. These criteria are slightly more restrictive than those adopted by Megeath et al. (2004) in order to attempt to create as "pure" a set of Class II objects as pos- sible. With our selection criteria, we may lose reddened Class II objects, but assuming that X-ray properties of Class II stars are independent of their reddening, our sample should be representative of the Class II population.

Using this criterion, we find 148 Class II stars with NIR photometry given in Table 4, analogous to Table 2; 130 of these objects (about 88\%) are X-ray detected. One of them, the COUP source 1401, is listed by Getman et al. (2005b) as a flaring source and classified as an Orion Molecular Core (OMC) member, whereas another Class II object, COUP source 751, was classified by the same work as an extragalactic object. Among the 130 X-ray-detected Class II stars with a counterpart in the H97 cata$\log , 83$ have a measure of the equivalent width of the Ca II 28542 line $W(\mathrm{Ca}$ II $)$. Among these, 28 have $W(\mathrm{Ca}$ II $)<-1$, which is the limit used by Flaccomio et al. (2003b) to classify strong accretors. Therefore, we have about 55 objects (i.e., $\sim 66 \%$ ) that have an NIR bright inner disk but relatively low accretion rate. Of the 148 Class II stars we have identified, we consider in the 


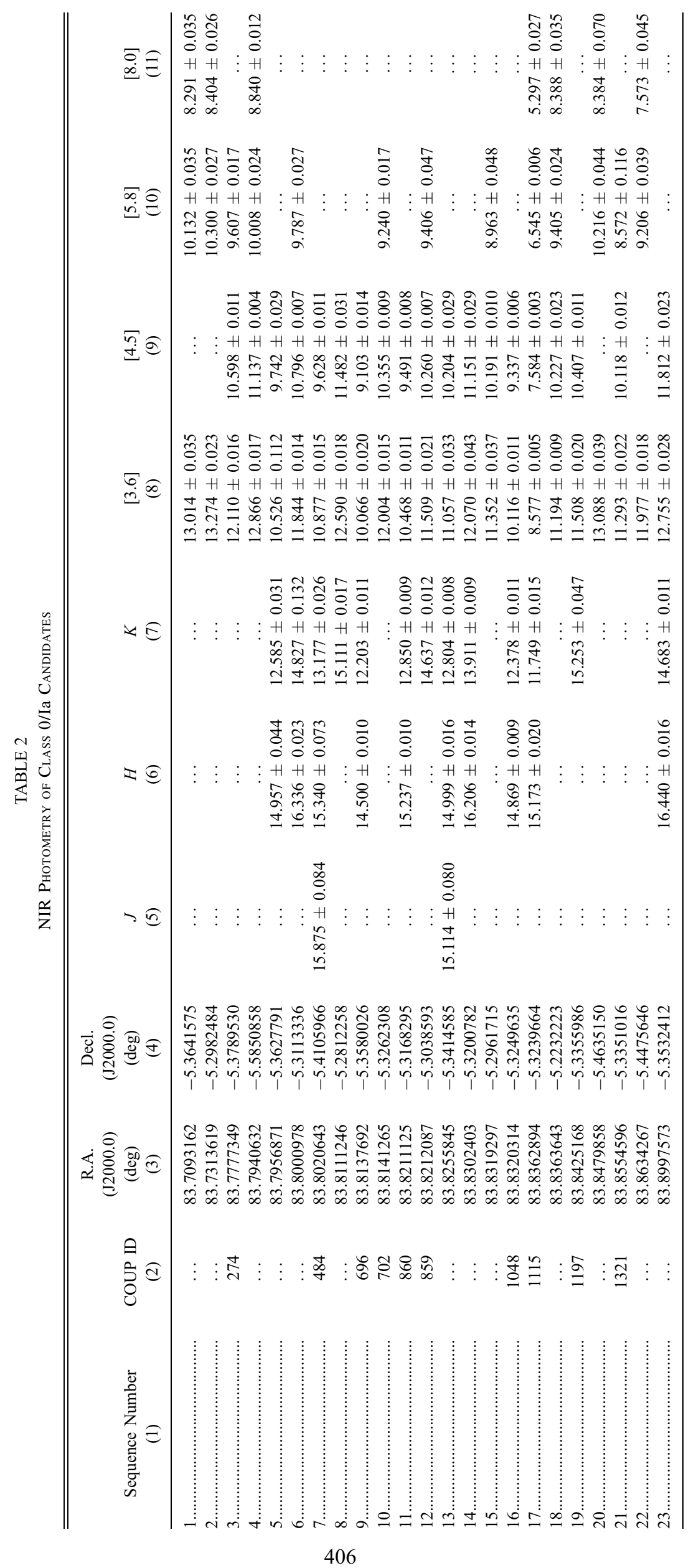



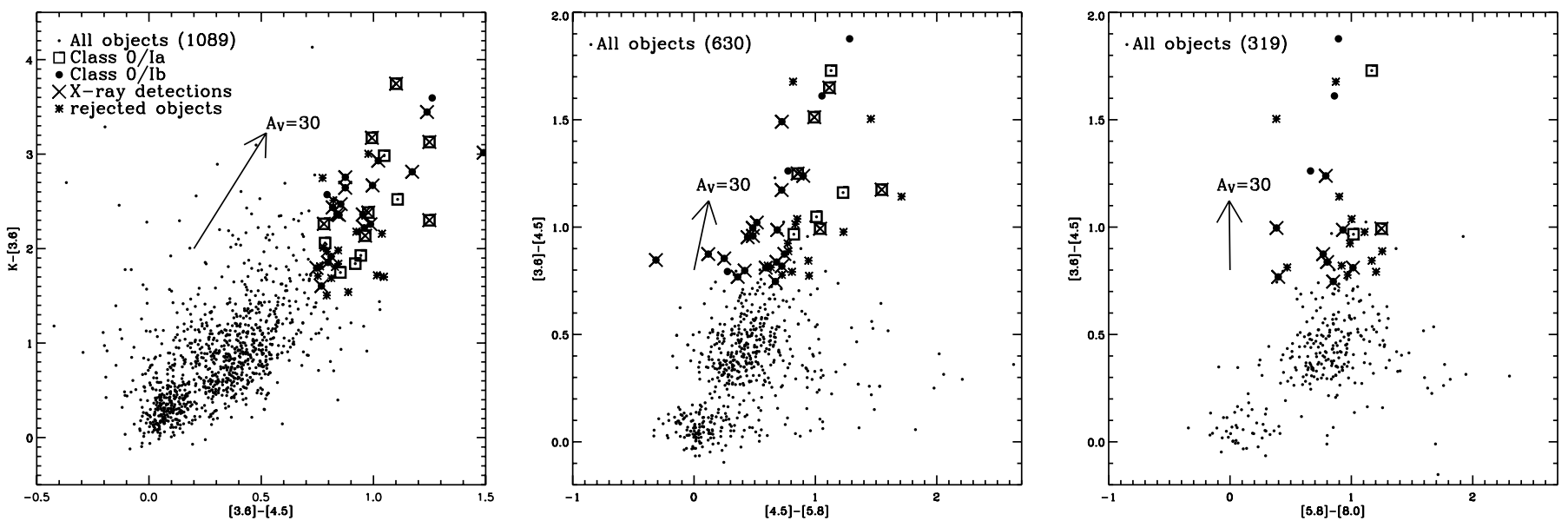

FIg. 3.- CCD of the stars in the COUP FOV (dots) created using the $K$ and the Spitzer IRAC magnitudes. The total number of objects in each diagram is reported in the three panels. Squares and large filled circles represent the Class 0 -Ia and $0-$ Ib objects, respectively, while crosses indicate those with an X-ray counterpart; asterisks indicate objects rejected because they have an optical counterpart. The reddening vectors computed using the IR reddening law given in Flaherty et al. (2007) for Orion A are also plotted.

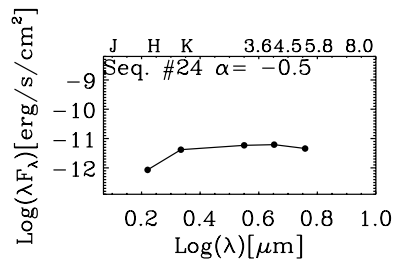

"ू్्र

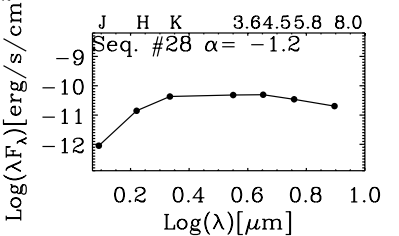

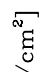

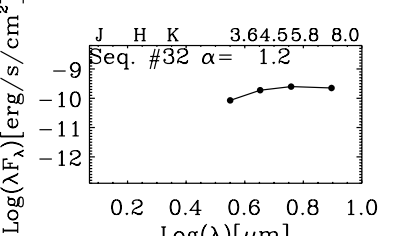

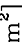

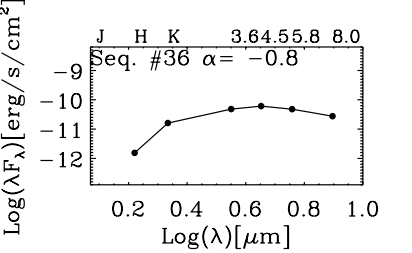

${ }^{\pi} g$
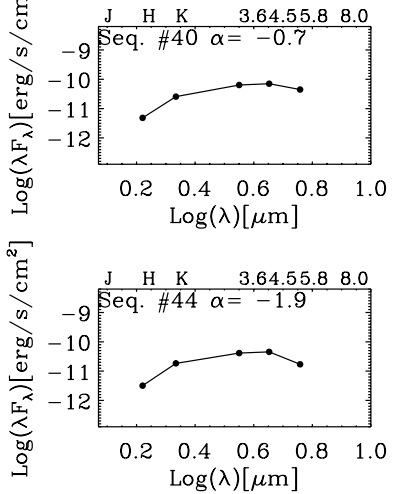
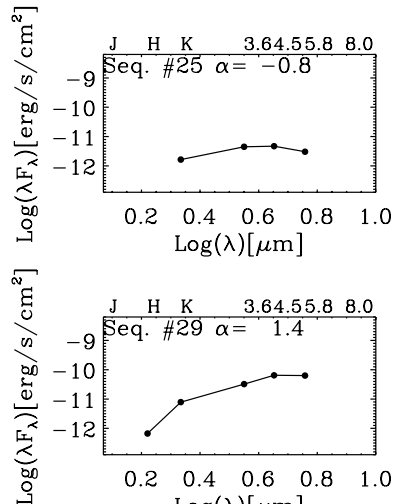

$\log (\lambda)[\mu \mathrm{m}]$

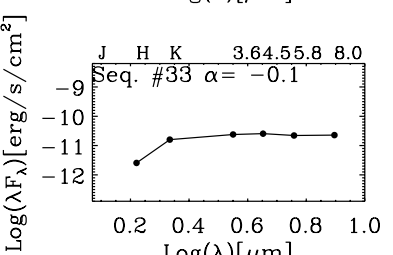

$\log (\lambda)[\mu \mathrm{m}]$

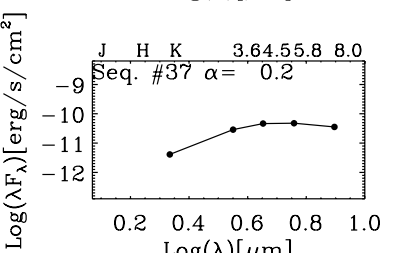

$\log (\lambda)[\mu \mathrm{m}]$
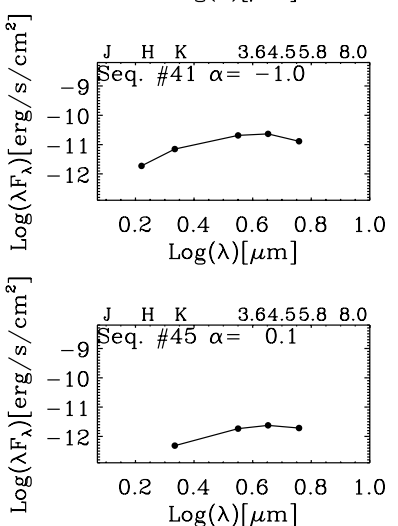
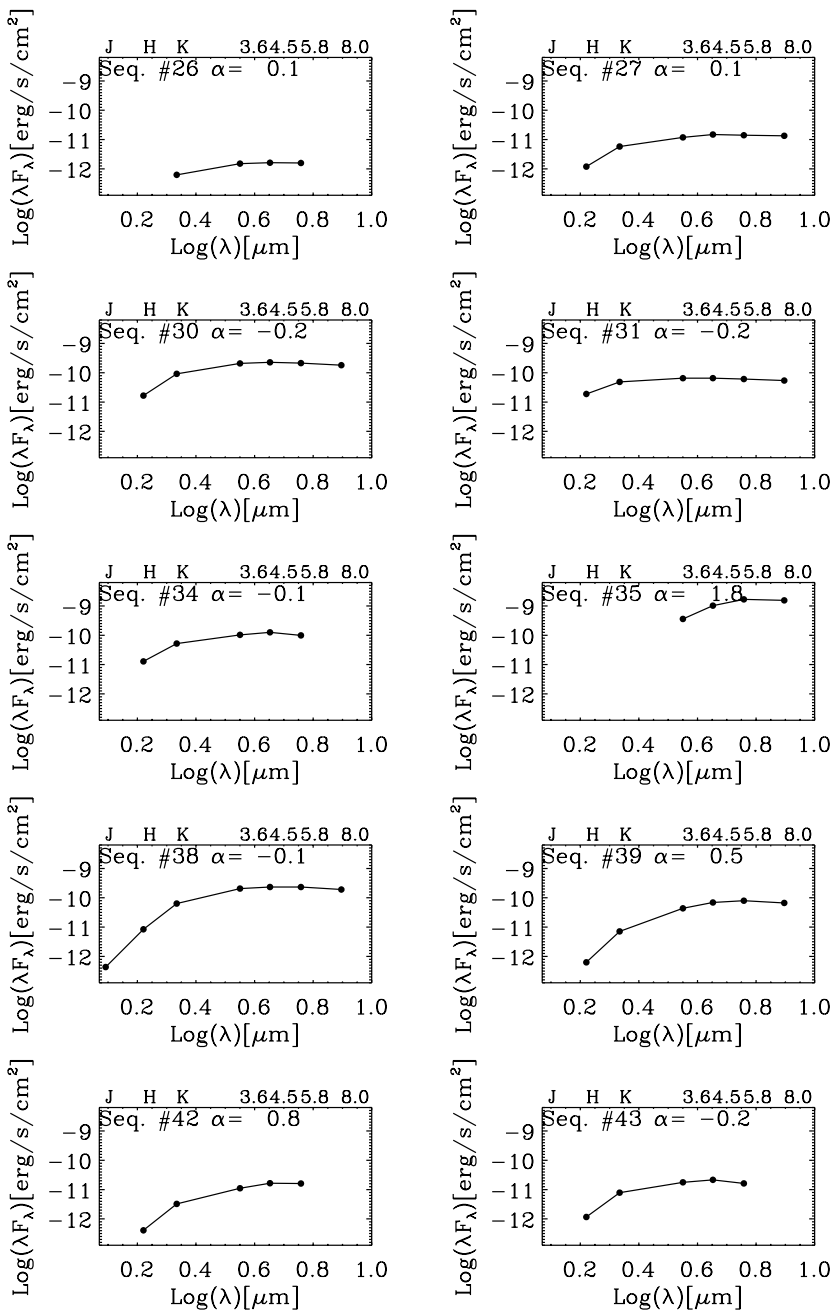

FIG. 4.-SED of the 22 Class $0-$ Ib candidate objects with a rising SED from $K$ to $4.5 \mu \mathrm{m}$. The sequential number in our catalog and the spectral index computed using the available IRAC magnitudes are also indicated. 


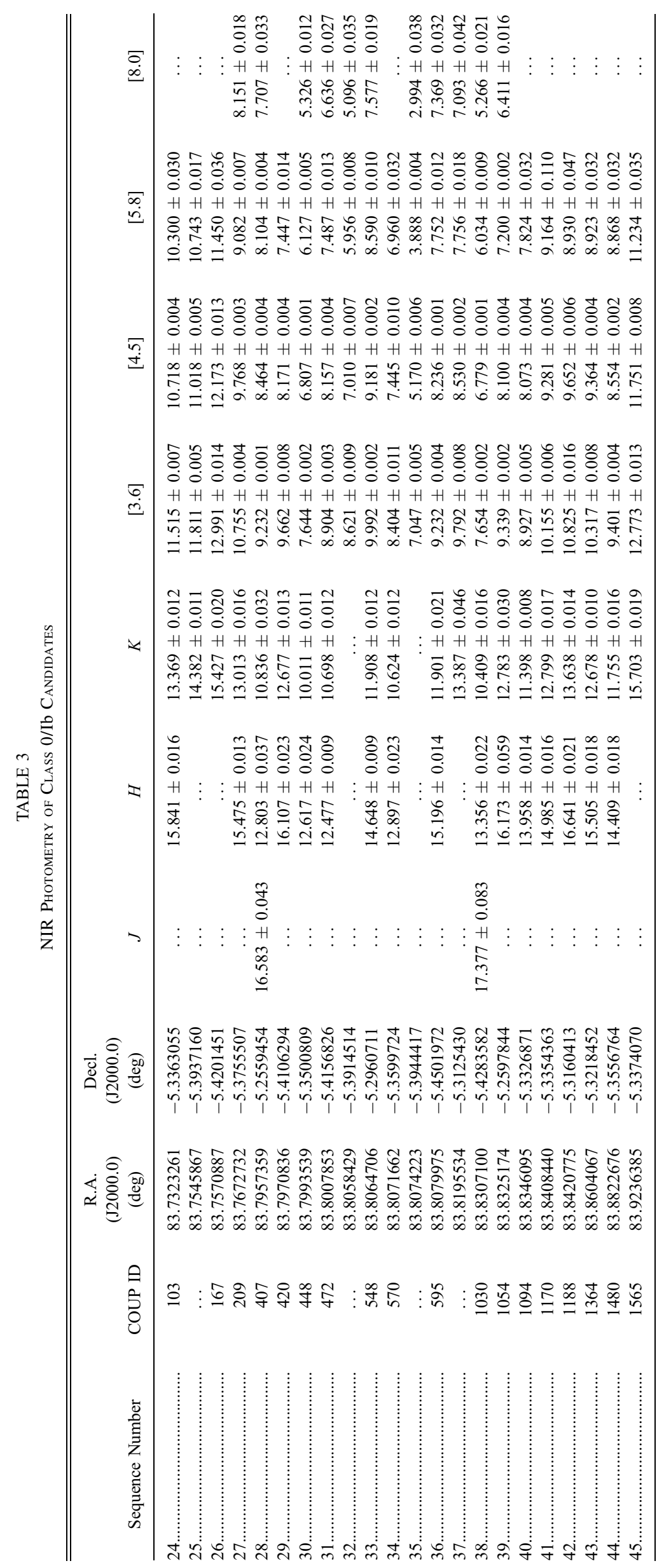



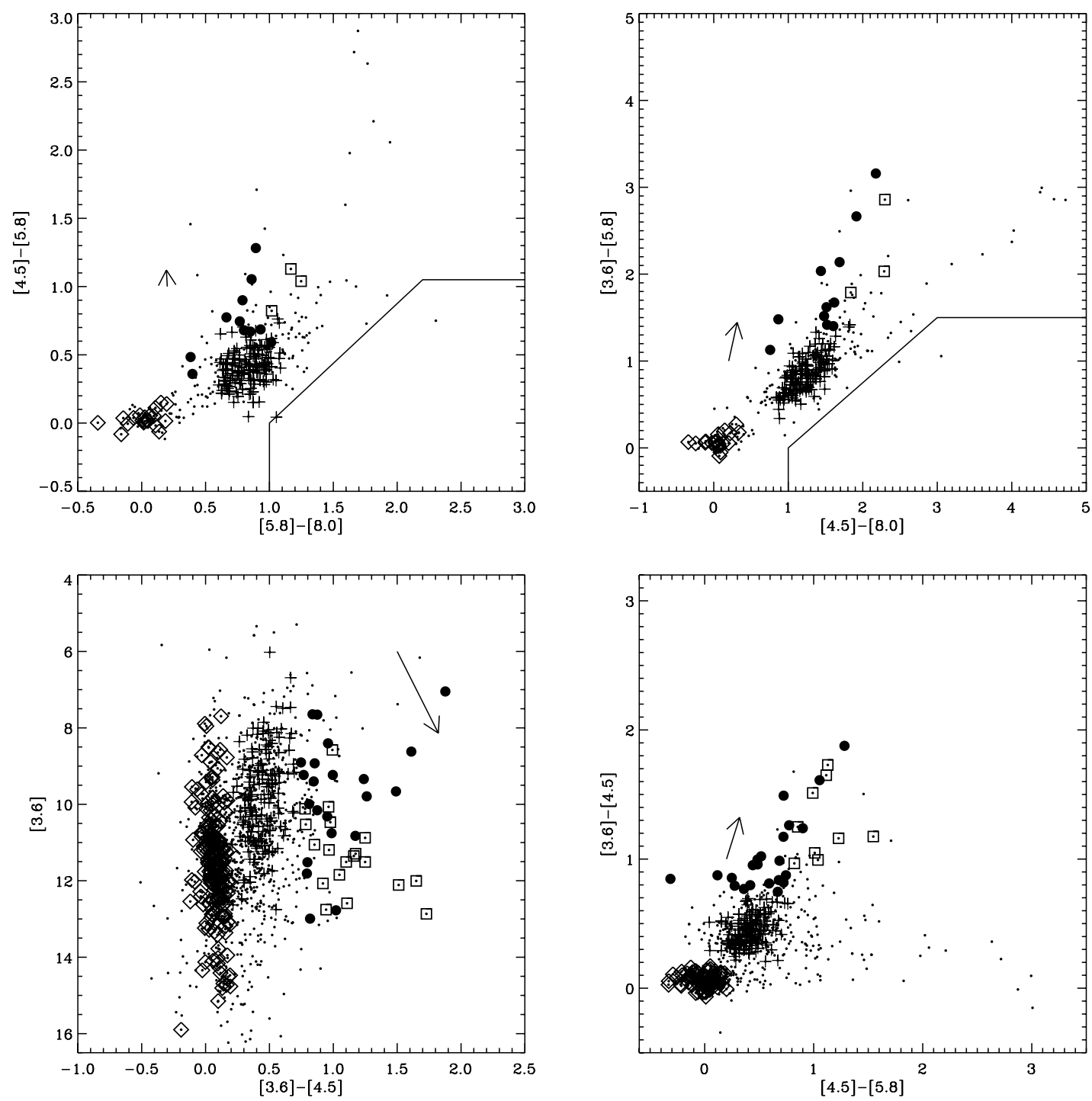

FIG. 5.-IRAC CCD and color-magnitude diagrams of the different populations in the ONC. Dots indicate all the stars in the COUP FOV with detections at the bands used in each panel. Squares and large filled circles are the Class $0-\mathrm{Ia}$ and $0-\mathrm{Ib}$ objects, respectively, plus signs are the stars classified as Class II, and diamonds are the Class III stars. The reddening vector corresponding to $A_{V}=30$, computed using the Flaherty et al. (2007) reddening law, is plotted in each panel. The solid lines in the above panels separate the region dominated by YSOs from the region expected to be dominated by galaxies.
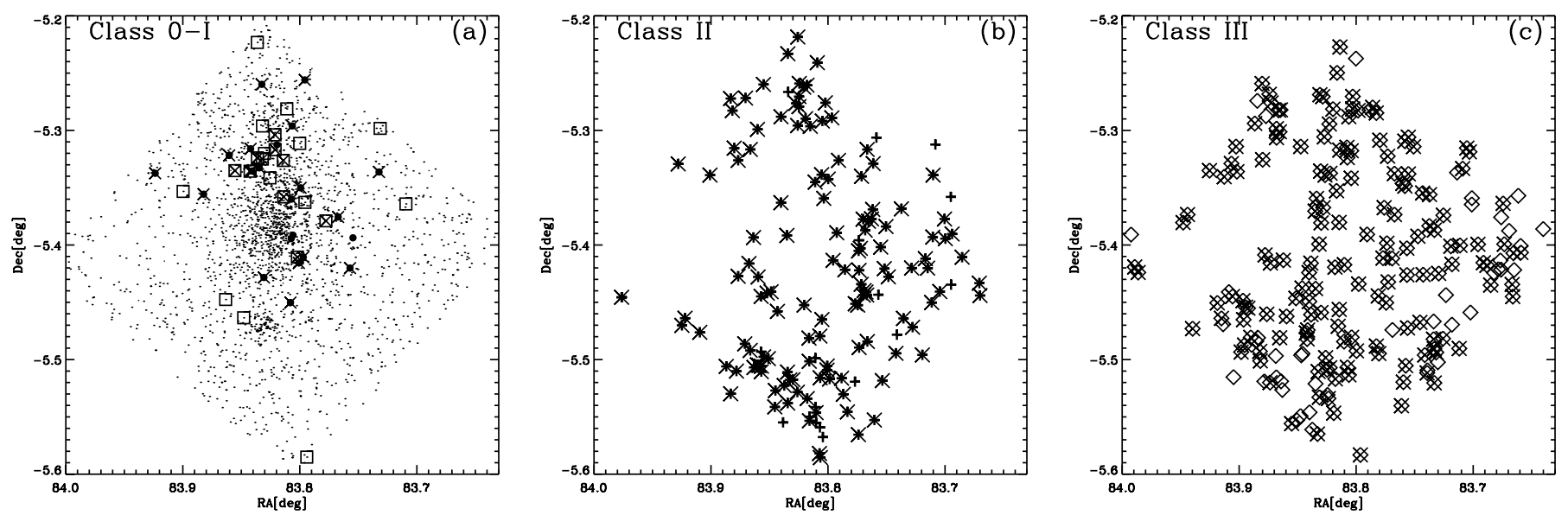

FIG. 6.- Spatial distribution of all the objects in our catalog within the COUP FOV that have been detected in at least two of the UBVIJHK and IRAC bands. Squares and large filled circles indicate Class 0 -Ia and $0-$ Ib objects, respectively, plus signs the Class II stars, and diamonds the Class III objects. In all the panels, X-ray-detected objects are indicated with a cross. 


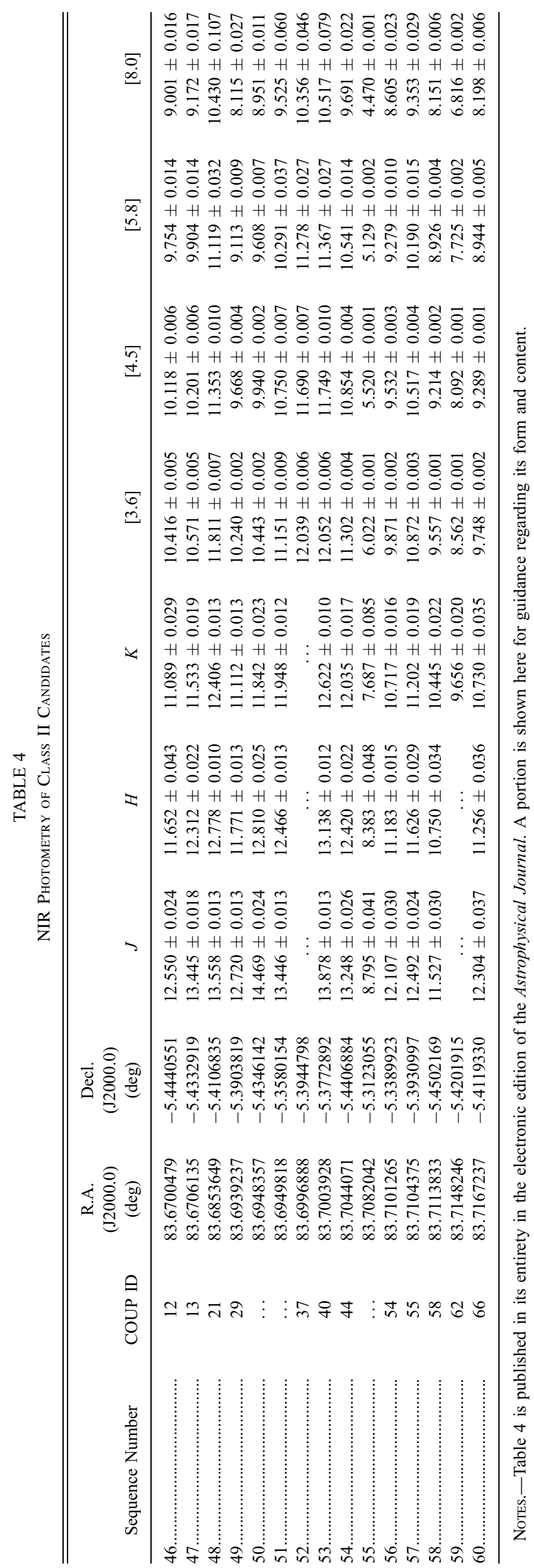


following analysis only the $87 \mathrm{X}$-ray-detected and the $9 \mathrm{X}$-rayundetected stars for which masses are known (H97; Getman et al. 2005a). We retain only these stars in order to study the X-ray properties as a function of stellar mass.

\subsection{Class III Stars}

Class III objects are PMS stars that do not show IR excesses and therefore were selected as those having IRAC colors near zero. In order to include all the stars not detected in the longer wavelength IRAC bands, we selected all the stars with $K-[3.6]<$ 0.5 and rejected those with $[3.6]-[4.5]>0.2$ or $[4.5]-[5.8]>$ 0.2 or $[5.8]-[8.0]>0.2$, when the latter colors were available, i.e., objects with IR excesses. Stars undetected in the IRAC bands at wavelengths longer than $3.6 \mu \mathrm{m}$ were included since their nondetection in these bands is compatible with purely photospheric SEDs.

Using these criteria, we selected 205 objects, with photometry given in Table 5; 160 of them (about 80\%) have an X-ray counterpart. Among the latter, 150 are in the list of H97. In particular, 130 of them have a proper-motion membership probability larger than $90 \%, 6$ have a membership probability equal to 0 , and the other 14 have no membership probabilities. For the remaining 10 stars without a counterpart in the list of H97 X-ray detection is the only known membership criterion. We note that none of these 10 objects are either in the list of flaring or in that of nonflaring sources of Getman et al. (2005a). Therefore, the membership class for these objects remains uncertain.

Of the 45 objects without an X-ray detection, 18 have a counterpart in the H97 catalog, but only 5 of these have a propermotion membership probability larger than $90 \%$.

The remaining 40 objects without a membership criterion could be either Class III stars without X-ray activity or field stars. Of the 205 Class III stars initially selected, we consider the 103 $\mathrm{X}$-ray-detected stars for which the mass is known (Getman et al. 2005a) and with H97 membership probability larger than $90 \%$; in addition, from the sample of X-ray-undetected Class III candidates, we retain only the two objects with membership probability larger than $90 \%$ and for which a mass has been derived by $\mathrm{H} 97$.

Figure 5 shows the selected stars in the IRAC CCD and colormagnitude diagrams. These diagrams show that our samples of YSOs most likely do not include active galactic nuclei (AGNs) or star-forming galaxies; that is, they do not include objects falling in the wedge-shaped regions outlined in the top panels of Figure 5. Star-forming galaxies are usually dominated by polycyclic aromatic hydrocarbon (PAH) features in the 5.8 and $8.0 \mu \mathrm{m}$ and therefore have $[5.8]-[8.0]>1.0$ but $[3.6]-[4.5] \lesssim 0.3$, which corresponds to the regions indicated by solid lines in the top panels of Figure 5 (Gutermuth et al. 2008). AGNs, instead, have $[3.6]-[4.5]$ and $[5.8]-[8.0]$ colors very similar to the Class II YSOs but are typically much fainter than YSOs, having, for example, [4.5] magnitudes (Megeath et al. 2005) much fainter than those of the selected YSOs (see Fig. 5, bottom left panel).

\section{SPATIAL DISTRIBUTION}

The spatial distribution of YSOs within the molecular cloud from which they have formed may give important clues for understanding the star formation process. Using the classification defined in the previous section, we have analyzed the spatial distribution of the YSOs in the ONC, as shown in Figure $6 a$, where we plot the position of all 2590 objects in our catalog within the COUP FOV that have been detected in at least two of the UBVIJHK and IRAC bands and the Class $0-$ I candidates. Stars of Class II and III are plotted in Figures $6 b$ and $6 c$, respectively.
Figure 7 shows the velocity-integrated ${ }^{13} \mathrm{CO}$ map obtained by Bally et al. (1987) and the Spitzer IRAC image at $3.6 \mu \mathrm{m}$ where the different classes selected in this work have been overplotted. The upper green box indicates a $40^{\prime \prime} \times 50^{\prime \prime}$ region around the Becklin-Neugebauer object and the Kleinmann Nebula, also indicated as BN/KL (R.A.J2000.0 $=05^{\mathrm{h}} 35^{\mathrm{m}} 14.16^{\mathrm{s}}$, decl. ${ }_{\mathrm{J} 2000.0}=$ $\left.-05^{\circ} 22^{\prime} 21.5^{\prime \prime}\right)$. The lower green box indicates a $60^{\prime \prime} \times 75^{\prime \prime}$ region around the Orion Molecular Cloud 1 (OMC-1S; R.A.J2000.0 = $05^{\mathrm{h}} 35^{\mathrm{m}} 14.50^{\mathrm{s}}$, decl.J2000.0 $\left.=-05^{\circ} 25^{\prime} 49.0^{\prime \prime}\right)$. These boxes show the limits of the areas studied in Grosso et al. (2005).

Most of the Class $0-\mathrm{I}$ stars are concentrated in the central and northeast parts of the COUP FOV along a region running northsouth from the Trapezium that follows the north-south distribution of the ${ }^{13} \mathrm{CO}$ emission, i.e., of the dense star-forming gas. The distribution of our selected Class 0 -I objects is biased by our NIR observations, which are more sensitive toward the north of the molecular ridge where the nebular contribution is lower. The lack of Class $0-\mathrm{I}$ objects in the $\mathrm{BN} / \mathrm{KL}$ and $\mathrm{OMC}-1 \mathrm{~S}$ regions is due to the bright nebulosity, as is evident from the image at $3.6 \mu \mathrm{m}$, which causes a decrease of sensitivity in the central region. For this reason, the spatial distribution of our Class $0-\mathrm{I}$ objects cannot be directly compared with that obtained by Lada et al. $(2000,2004)$ although in fact the two spatial distributions are quite similar.

Figures 6 and 7 show the same structure already found in Feigelson et al. (2005) using the lightly X-ray-absorbed stars: they interpreted this distribution as due to a process of violent relaxation. Note that the latter study examined the spatial distributions stratified by absorption, while here the stars are stratified by individual YSO evolutionary classification. The features of the spatial distribution visible in Figures 6 and 7 include the OMC-1S core, BN/KL core, and northern extension, which are part of the larger filament along the middle of the Orion A cloud, and finally the east-west asymmetry that in Feigelson et al. (2005) was attributed to nonequilibrium dynamics. The latter only shows up in Class III systems.

The spatial distributions of Class II and III stars are also in agreement with the spatial distribution found by H97, who, using D'Antona \& Mazzitelli (1994) isochrone ages, found that youngest stars are clustered toward the cluster center while slightly older stars are more widely distributed. The elongated asymmetric distribution of our candidate Class $0-$ I stars and the large-scale EW asymmetry are consistent with the distribution derived by Hillenbrand \& Hartmann (1998), who concluded that the structure of the $\mathrm{ONC}$ is more the result of the different environmental conditions rather than the result of dynamical evolution.

There is a lower percentage of Class II objects in the central region, but this could be due to the reliance on the $8.0 \mu \mathrm{m}$ detections for the selection of Class II stars. Our spatial distributions are, therefore, strongly dependent on the sensitivity of our NIR data, which is quite limited toward the central region, where the contamination by the bright $\mathrm{OB}$ stars and nebular emission is very high.

\section{EVOLUTION OF X-RAY ACTIVITY}

The very deep X-ray COUP data together with the relatively large sample of Class 0-I objects selected with the Spitzer observations allow us, for the first time, to analyze and compare, in a statistically significant way, the X-ray properties of the YSOs in their initial protostellar phase with those of more evolved PMS stars.

As already mentioned in $\S 3$, we find that the fraction of Class 0 Ia objects detected in the X-rays is almost $40 \%(10 / 23)$, that of 


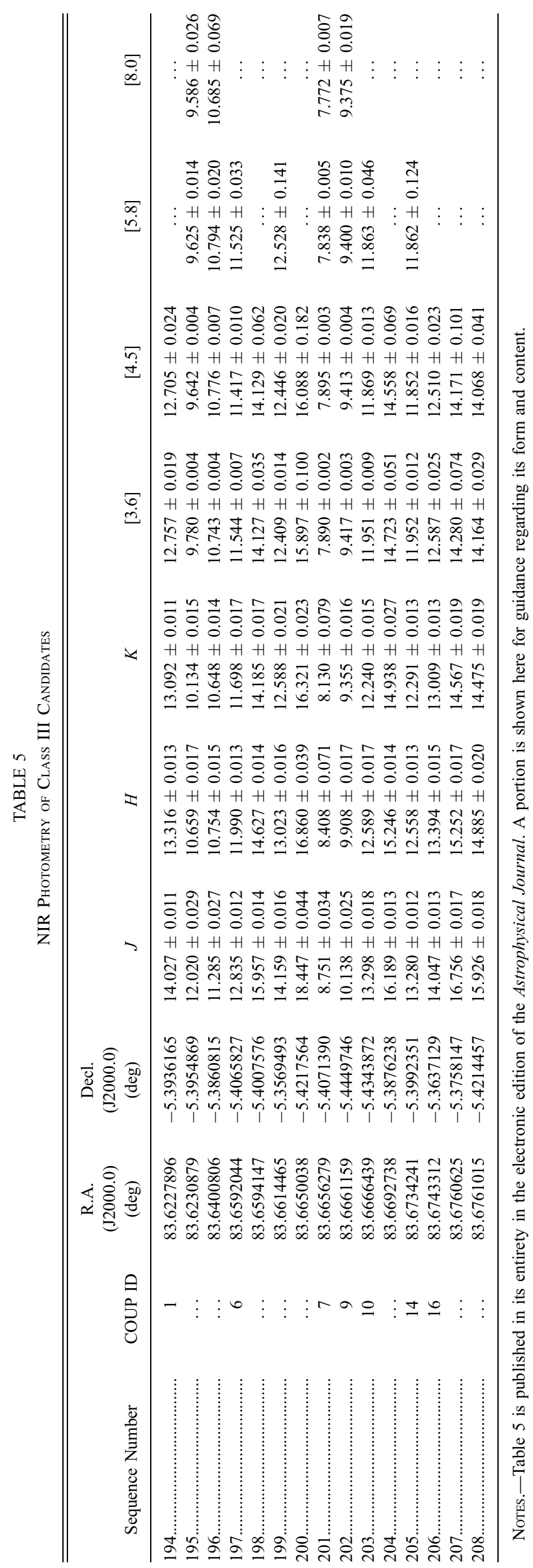




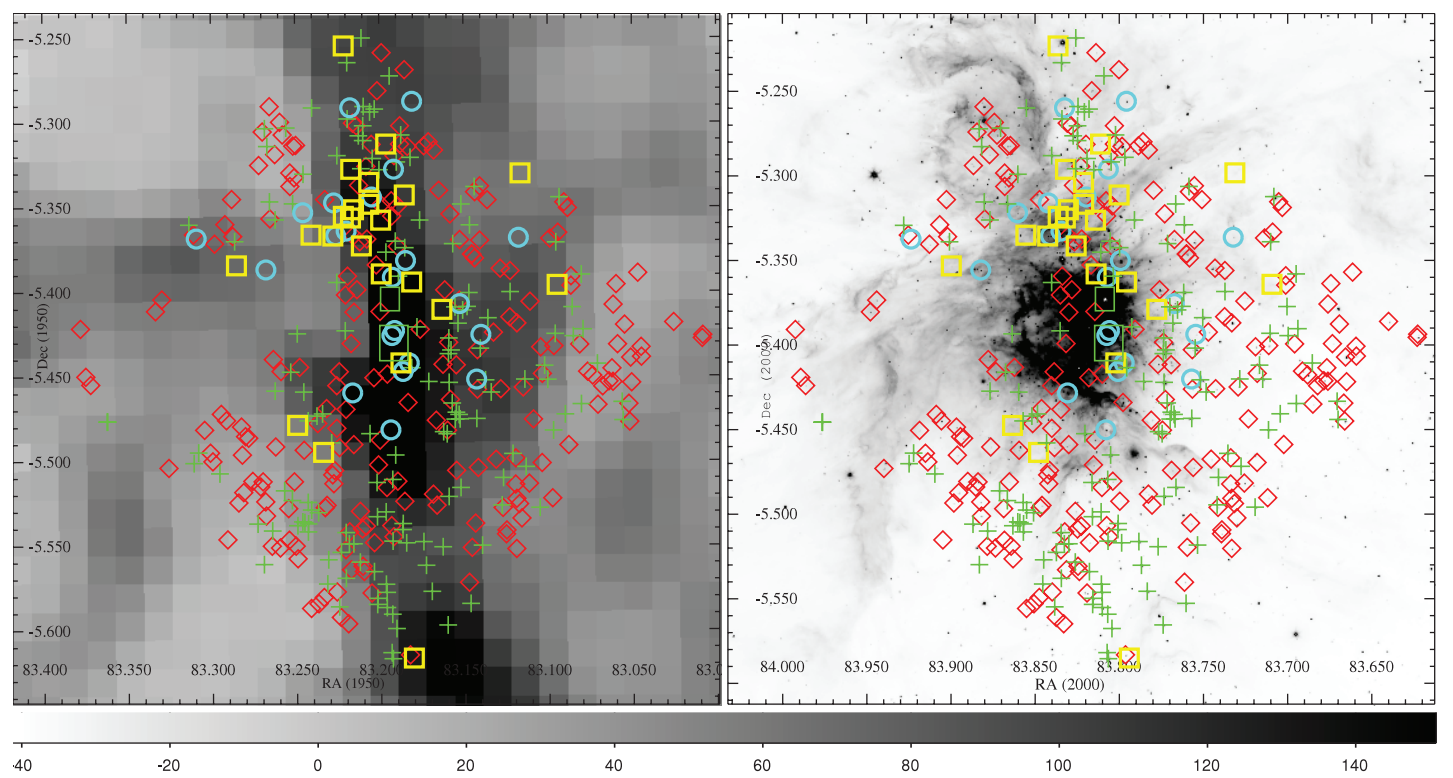

FIG. 7.- Velocity-integrated ${ }^{13} \mathrm{CO}$ emissivity from Bally et al. (1987) in the region around the Trapezium cluster (left) and Spitzer IRAC image at $3.6 \mu \mathrm{m}$ in the same region (right). Both images are shown with inverted color map. Yellow squares and light blue circles indicate Class $0-$ Ia and $0-$ Ib objects, respectively, green plus signs are Class II stars, and red diamonds are Class III stars. The $40^{\prime \prime} \times 50^{\prime \prime}$ upper green box indicates the BN/KL region, while the $60^{\prime \prime} \times 75^{\prime \prime}$ bottom green box indicates the Orion Molecular Cloud 1 South (OMC-1S).

Class $0-\mathrm{Ib}$ and III is about $80 \%(18 / 22$ and $160 / 205$, respectively), while that of Class II objects is about $88 \%$ (130/148). The analysis described in this section aims at understanding whether the X-ray emission can be related to the IR properties of YSOs, which we take as signposts for the different phases of protostellar and PMS evolution.

\subsection{X-Ray Luminosities}

We use the results of the spectral analysis presented by Getman et al. (2005a) to compare the X-ray properties of the different populations of YSOs in the ONC. For the X-ray luminosities of detected stars we adopt the absorption-corrected luminosities in the total $0.5-8.0 \mathrm{keV}$ band, $L_{t, c}$, from Table 8 of Getman et al. (2005a).

In order to compare unbiased luminosity functions of the different classes, we derive upper limits to the X-ray luminosities of the X-ray-undetected objects. Upper limits to the photon count rates of the $13+4$ undetected Class $0-\mathrm{Ia}$ and $0-\mathrm{Ib}$ objects were calculated with PWDetect (Damiani et al. 1997a, 1997b) consistently with the source detection procedure of the other COUP sources (Getman et al. 2005a), adopting a detection threshold significance of $5 \sigma$. The upper limits for the nine undetected Class II stars and for the two undetected Class III stars were taken from Table 11 of Getman et al. (2005a).

In order to convert upper limits to the count rates into unabsorbed X-ray luminosities, we derive an extinction-dependent conversion factor. The CCDs in Figure 3 indicate that X-raydetected and X-ray-undetected Class $0-\mathrm{I}$ objects have similar reddening. We therefore computed the conversion factors of the X-ray-detected Class 0-I objects as the ratio between the unabsorbed X-ray luminosities $\left(L_{t, c}\right)$ and the count rates taking into account the point-spread function (PSF) fraction within the extracted area and the effective exposure time at the source position (Getman et al. 2005a), and we plotted these values as a function of the $N_{\mathrm{H}}{ }^{9}$ values. We assume for the X-ray-undetected Class 0-I

\footnotetext{
9 The $\mathrm{H}$ column densities were derived by Getman et al. (2005a) from the spectral analysis of the COUP sources.
}

objects a conversion factor computed as the median value of the conversion factors of the X-ray-detected Class $0-\mathrm{I}$ objects, which corresponds for these stars to $N_{\mathrm{H}} \simeq 4.5 \times 10^{22} \mathrm{~cm}^{-2}$. However, in order to check whether our results are robust with respect to the assumed $N_{\mathrm{H}}$ of undetected objects, we also considered the conversion factors corresponding to the highest and minimum conversion factors of the X-ray-detected Class 0-I objects. ${ }^{10}$ We used the same procedure to estimate the upper limits to the $\mathrm{X}$-ray luminosities in the $2.0-8.0 \mathrm{keV}$ hard band.

The X-ray properties of the Class 0-I COUP X-ray sources, taken from Getman et al. (2005a), are given in Table 6. We provide sequential numbers from our catalog, sequential COUP numbers, background-corrected counts in the $0.5-8.0 \mathrm{keV}$ band, the fractions of the PSF within the source extraction areas, effective exposure times, the hydrogen column densities obtained from the spectral fit, plasma temperatures, emission measures, and finally the absorption-corrected X-ray luminosities in the 2.0$8.0 \mathrm{keV}$ hard band, $L_{h, c}$, and in the $0.5-8.0 \mathrm{keV}$ total band, $L_{t, c}$.

Values computed for the X-ray-undetected Class 0 -I stars are given in Table 7. We list sequential numbers from our catalog, the celestial coordinates from the Spitzer catalog, upper limits to the source counts computed with PWDetect in the hard and total bands, the effective Chandra exposure time at the object positions, and finally, the upper limits to the unabsorbed X-ray luminosities in the hard and total energy bands.

X-ray properties of X-ray-detected Class II and III stars, taken from Getman et al. (2005a), are given in Tables 8 and 9, where column names are analogous to those given in Table 6.

The X-ray luminosities of X-ray-undetected Class II and III objects were derived, as for Class $0-\mathrm{I}$ stars, using the median conversion factor of the X-ray-detected stars in the same class. This assumption is based on the fact that the NIR colors of the X-ray-undetected Class II stars indicate that these objects are not more absorbed than those detected with COUP. In addition,

10 We used the median conversion factor to derive the X-ray luminosity $\left(\log L_{t, c}=28.52 \mathrm{ergs} \mathrm{s}^{-1}\right)$ of the COUP source 1197 for which Getman et al. (2005a) did not perform a spectral fit because of the low counting statistics. 
TABLE 6

X-Ray Photometry of the Class 0/I Candidates Taken from Getman et al. (2005a)

\begin{tabular}{|c|c|c|c|c|c|c|c|c|c|}
\hline Sequence Number & COUP ID & Net Counts & PSF Fraction & $\begin{array}{c}\text { Exposure } \\
(\mathrm{ks})\end{array}$ & $\begin{array}{l}\log N_{\mathrm{H}} \\
\left(\mathrm{cm}^{-2}\right)\end{array}$ & $\begin{array}{c}k T_{1} \\
(\mathrm{keV})\end{array}$ & $\begin{array}{c}\log \mathrm{EM}_{1} \\
\left(\mathrm{~cm}^{-3}\right)\end{array}$ & $\begin{array}{c}\log L_{h, c} \\
\left(\operatorname{ergs~s}^{-1}\right)\end{array}$ & $\begin{array}{c}\log L_{t, c} \\
\left(\operatorname{ergs~s}^{-1}\right)\end{array}$ \\
\hline $3 \ldots$ & 274 & 176 & 0.86 & 762.20 & $22.56 \pm 0.06$ & $15.00 \pm 15.00$ & $52.28 \pm 0.06$ & 29.28 & 29.42 \\
\hline $7 \ldots \ldots$ & 484 & 54 & 0.86 & 783.40 & $22.54 \pm 0.14$ & $2.85 \pm 1.67$ & $51.96 \pm 0.31$ & 28.67 & 29.00 \\
\hline $9 \ldots \ldots$ & 696 & 27 & 0.87 & 825.80 & $22.16 \pm 0.28$ & $9.06 \pm 15.00$ & $51.19 \pm 0.31$ & 28.19 & 28.38 \\
\hline $10 \ldots \ldots$ & 702 & 79 & 0.86 & 801.10 & $22.31 \pm 0.17$ & $3.25 \pm 1.71$ & $51.86 \pm 0.27$ & 28.63 & 28.93 \\
\hline $11 \ldots \ldots$. & 860 & 185 & 0.87 & 792.20 & $22.60 \pm 0.12$ & $5.73 \pm 4.09$ & $52.34 \pm 0.20$ & 29.27 & 29.49 \\
\hline $12 \ldots \ldots$. & 859 & 481 & 0.87 & 751.60 & $22.77 \pm 0.05$ & $1.90 \pm 0.22$ & $53.28 \pm 0.12$ & 29.78 & 30.24 \\
\hline $16 \ldots \ldots \ldots \ldots \ldots \ldots \ldots \ldots \ldots \ldots \ldots \ldots \ldots \ldots \ldots \ldots$ & 1048 & 162 & 0.87 & 795.80 & $22.68 \pm 0.08$ & $2.98 \pm 0.84$ & $52.51 \pm 0.19$ & 29.24 & 29.56 \\
\hline $17 \ldots \ldots \ldots \ldots \ldots \ldots \ldots \ldots \ldots \ldots \ldots \ldots \ldots$ & 1115 & 27 & 0.86 & 794.00 & $22.83 \pm 0.19$ & $1.13 \pm 0.70$ & $52.43 \pm 1.00$ & 28.56 & 29.37 \\
\hline $19 \ldots$. & 1197 & 15 & 0.87 & 799.30 & $\ldots$ & $\ldots$ & $\ldots$ & $\ldots$ & $\ldots$ \\
\hline $21 \ldots \ldots \ldots \ldots \ldots \ldots \ldots$ & 1321 & 184 & 0.87 & 714.40 & $23.11 \pm 0.09$ & $4.51 \pm 2.98$ & $52.82 \pm 0.29$ & 29.69 & 29.94 \\
\hline 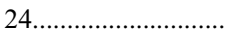 & 103 & 58 & 0.87 & 783.40 & $22.63 \pm 0.13$ & $0.95 \pm 0.35$ & $52.64 \pm 1.00$ & 28.61 & 29.60 \\
\hline $26 \ldots \ldots$ & 167 & 88 & 0.86 & 739.20 & $22.73 \pm 0.10$ & $1.16 \pm 0.24$ & $52.79 \pm 1.00$ & 28.94 & 29.72 \\
\hline 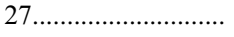 & 209 & 97 & 0.87 & 824.10 & $22.37 \pm 0.13$ & $4.86 \pm 3.60$ & $51.90 \pm 0.22$ & 28.80 & 29.03 \\
\hline $28 \ldots \ldots \ldots \ldots \ldots \ldots \ldots \ldots \ldots \ldots \ldots \ldots \ldots \ldots \ldots$ & 407 & 479 & 0.87 & 737.40 & $22.63 \pm 0.07$ & $3.61 \pm 0.99$ & $52.91 \pm 0.16$ & 29.71 & 30.00 \\
\hline $29 \ldots \ldots \ldots \ldots \ldots \ldots \ldots \ldots \ldots$ & 420 & 65 & 0.86 & 838.20 & $22.29 \pm 0.13$ & $3.45 \pm 2.65$ & $51.70 \pm 0.26$ & 28.48 & 28.78 \\
\hline 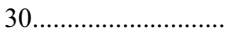 & 448 & 2249 & 0.86 & 820.50 & $22.52 \pm 0.02$ & $2.24 \pm 0.12$ & $53.64 \pm 0.05$ & 30.24 & 30.64 \\
\hline $31 \ldots \ldots \ldots \ldots \ldots \ldots \ldots \ldots \ldots \ldots \ldots \ldots \ldots$ & 472 & 501 & 0.86 & 456.20 & $22.55 \pm 0.05$ & $2.80 \pm 0.38$ & $53.18 \pm 0.10$ & 29.88 & 30.21 \\
\hline 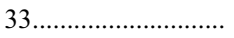 & 548 & 2315 & 0.87 & 774.50 & $22.54 \pm 0.02$ & $3.06 \pm 0.25$ & $53.59 \pm 0.04$ & 30.33 & 30.65 \\
\hline 34......................... & 570 & 4070 & 0.87 & 829.40 & $22.64 \pm 0.02$ & $3.35 \pm 0.22$ & $53.86 \pm 0.03$ & 30.63 & 30.93 \\
\hline $36 \ldots \ldots \ldots \ldots \ldots \ldots \ldots \ldots \ldots \ldots \ldots \ldots \ldots$ & 595 & 624 & 0.87 & 779.80 & $22.69 \pm 0.04$ & $2.31 \pm 0.18$ & $53.23 \pm 0.10$ & 29.84 & 30.23 \\
\hline $38 \ldots \ldots \ldots \ldots \ldots \ldots \ldots \ldots \ldots \ldots \ldots \ldots \ldots \ldots \ldots$ & 1030 & 3616 & 0.87 & 749.80 & $22.98 \pm 0.02$ & $15.00 \pm 1.00$ & $53.89 \pm 0.03$ & 30.88 & 31.01 \\
\hline 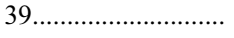 & 1054 & 1630 & 0.88 & 707.30 & $22.64 \pm 0.02$ & $2.59 \pm 0.25$ & $53.62 \pm 0.07$ & 30.28 & 30.64 \\
\hline $40 \ldots \ldots \ldots \ldots \ldots \ldots \ldots \ldots \ldots \ldots \ldots \ldots \ldots \ldots \ldots \ldots$ & 1094 & 214 & 0.87 & 801.10 & $22.61 \pm 0.07$ & $2.79 \pm 0.55$ & $52.60 \pm 0.17$ & 29.30 & 29.64 \\
\hline $41 \ldots \ldots \ldots \ldots \ldots \ldots \ldots \ldots \ldots$ & 1170 & 150 & 0.87 & 799.30 & $22.61 \pm 0.08$ & $1.03 \pm 0.22$ & $52.96 \pm 1.00$ & 29.02 & 29.91 \\
\hline $42 \ldots \ldots \ldots \ldots \ldots \ldots \ldots \ldots \ldots \ldots \ldots \ldots \ldots$ & 1188 & 232 & 0.87 & 686.10 & $22.73 \pm 0.04$ & $1.70 \pm 0.27$ & $53.10 \pm 0.16$ & 29.54 & 30.05 \\
\hline $43 \ldots \ldots \ldots \ldots \ldots \ldots \ldots \ldots \ldots$ & 1364 & 501 & 0.88 & 779.80 & $22.70 \pm 0.03$ & $4.30 \pm 1.02$ & $52.94 \pm 0.11$ & 29.80 & 30.05 \\
\hline 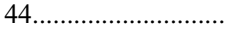 & 1480 & 914 & 0.86 & 684.40 & $22.69 \pm 0.03$ & $2.10 \pm 0.18$ & $53.51 \pm 0.08$ & 30.07 & 30.50 \\
\hline 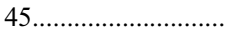 & 1565 & 385 & 0.88 & 746.20 & $22.52 \pm 0.05$ & $2.50 \pm 0.40$ & $52.85 \pm 0.12$ & 29.50 & 29.86 \\
\hline
\end{tabular}

Note.-A description of the columns is given in $\S 5.1$.

the conversion factors corresponding to the lowest and highest observed $N_{\mathrm{H}}$ values were also considered $\left(10^{21}\right.$ and $4.0 \times 10^{22} \mathrm{~cm}^{-2}$ for the Class II stars and $0.5 \times 10^{21}$ and $2.0 \times 10^{22} \mathrm{~cm}^{-2}$ for the Class III stars). Values computed for the X-ray-undetected Class II and III stars are given in Table 10, analogous to Table 7.

\subsection{X-Ray Luminosity Functions}

We now compare the XLFs of the stars of the four considered NIR classes. In order to take into account nondetections, XLFs were derived using the Kaplan-Meier maximum likelihood

TABLE 7

Upper Limits of the X-Ray-undetected Class 0/I Candidates

\begin{tabular}{|c|c|c|c|c|c|c|c|c|c|c|c|}
\hline \multirow[b]{2}{*}{ Sequence Number } & \multirow{2}{*}{$\begin{array}{c}\text { R.A. } \\
\text { (J2000.0) } \\
(\mathrm{deg})\end{array}$} & \multirow{2}{*}{$\begin{array}{c}\text { DECL. } \\
(\mathrm{J} 2000.0) \\
(\mathrm{deg})\end{array}$} & \multicolumn{2}{|c|}{ Counts } & \multirow{2}{*}{$\begin{array}{c}\text { EXPOSURE } \\
(\mathrm{ks})\end{array}$} & \multirow{2}{*}{$\begin{array}{c}\log L_{h, c}^{1} \\
\left(\operatorname{ergs~s}^{-1}\right)\end{array}$} & \multirow{2}{*}{$\begin{array}{c}\log L_{h, c}^{2} \\
\left(\operatorname{ergs~s}^{-1}\right)\end{array}$} & \multirow{2}{*}{$\begin{array}{c}\log L_{h, c}^{3} \\
\left(\operatorname{ergs~} \mathrm{s}^{-1}\right)\end{array}$} & \multirow{2}{*}{$\begin{array}{c}\log L_{t, c}^{1} \\
\left(\operatorname{ergs~s}^{-1}\right)\end{array}$} & \multirow{2}{*}{$\begin{array}{c}\log L_{t, c}^{2} \\
\left(\operatorname{ergs~s}^{-1}\right)\end{array}$} & \multirow{2}{*}{$\begin{array}{c}\log L_{t, c}^{3} \\
\left(\text { ergs s }{ }^{-1}\right)\end{array}$} \\
\hline & & & Hard & Total & & & & & & & \\
\hline .......... & 83.70931200 & -5.36415800 & 26.38 & 32.58 & 710.29 & 28.44 & 28.27 & 28.76 & 28.84 & 29.44 & 29.65 \\
\hline $2 \ldots \ldots \ldots \ldots \ldots \ldots \ldots \ldots \ldots \ldots \ldots \ldots \ldots \ldots$ & 83.73136100 & -5.29824800 & 69.32 & 86.29 & 739.17 & 28.84 & 28.67 & 29.16 & 29.25 & 29.85 & 30.05 \\
\hline $4 \ldots \ldots \ldots \ldots \ldots \ldots \ldots \ldots \ldots \ldots \ldots \ldots$ & 83.79406000 & -5.58508600 & 661.04 & 3605.31 & 681.67 & 29.85 & 29.69 & 30.18 & 30.90 & 31.50 & 31.71 \\
\hline $5 \ldots \ldots \ldots \ldots \ldots \ldots \ldots \ldots \ldots$ & 83.79568709 & -5.36277907 & 18.18 & 31.25 & 817.28 & 28.21 & 28.05 & 28.54 & 28.76 & 29.36 & 29.57 \\
\hline $6 \ldots \ldots \ldots \ldots \ldots \ldots$ & 83.80009500 & -5.31133400 & 21.00 & 26.16 & 781.40 & 28.29 & 28.13 & 28.62 & 28.70 & 29.30 & 29.51 \\
\hline$\ldots \ldots \ldots$ & 83.81112700 & -5.28122600 & 30.07 & 64.72 & 632.86 & 28.54 & 28.38 & 28.87 & 29.19 & 29.79 & 30.00 \\
\hline 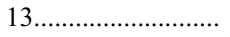 & 83.82558451 & -5.34145849 & 16.28 & 21.07 & 800.83 & 28.17 & 28.01 & 28.50 & 28.60 & 29.20 & 29.41 \\
\hline $14 \ldots \ldots \ldots \ldots \ldots \ldots \ldots \ldots$ & 83.83023800 & -5.32007800 & 19.08 & 22.96 & 782.92 & 28.25 & 28.09 & 28.58 & 28.65 & 29.25 & 29.45 \\
\hline $15 \ldots \ldots \ldots \ldots \ldots \ldots \ldots \ldots \ldots \ldots \ldots \ldots \ldots \ldots \ldots$ & 83.83193200 & -5.29617200 & 27.37 & 31.85 & 764.63 & 28.42 & 28.25 & 28.74 & 28.80 & 29.40 & 29.61 \\
\hline $18 \ldots \ldots \ldots \ldots \ldots \ldots \ldots \ldots \ldots$ & 83.83636500 & -5.22322200 & 2873.07 & 4004.38 & 698.35 & 30.48 & 30.31 & 30.80 & 30.94 & 31.54 & 31.75 \\
\hline $20 \ldots \ldots \ldots \ldots \ldots \ldots \ldots$ & 83.84798400 & -5.46351500 & 19.52 & 23.76 & 784.75 & 28.26 & 28.09 & 28.58 & 28.66 & 29.26 & 29.47 \\
\hline $22 \ldots \ldots \ldots \ldots \ldots \ldots \ldots \ldots \ldots \ldots \ldots$ & 83.86342600 & -5.44756500 & 16.24 & 20.93 & 732.72 & 28.21 & 28.04 & 28.53 & 28.63 & 29.23 & 29.44 \\
\hline $23 \ldots \ldots \ldots \ldots \ldots \ldots \ldots \ldots \ldots$ & 83.89975700 & -5.35324100 & 19.93 & 23.70 & 651.91 & 28.35 & 28.18 & 28.67 & 28.74 & 29.34 & 29.55 \\
\hline $25 \ldots \ldots \ldots \ldots \ldots \ldots \ldots \ldots$ & 83.75458500 & -5.39371600 & 14.13 & 18.14 & 762.24 & 28.16 & 27.94 & 28.37 & 28.56 & 29.15 & 29.36 \\
\hline $32 \ldots \ldots \ldots \ldots \ldots \ldots \ldots \ldots \ldots \ldots \ldots \ldots$ & 83.80584286 & -5.39145145 & 14.85 & 19.82 & 346.70 & 28.52 & 28.30 & 28.73 & 28.94 & 29.54 & 29.74 \\
\hline $35 \ldots \ldots \ldots \ldots \ldots \ldots \ldots \ldots \ldots \ldots \ldots$ & 83.80742228 & -5.39444168 & 14.63 & 22.59 & 336.57 & 28.53 & 28.31 & 28.74 & 29.01 & 29.60 & 29.81 \\
\hline 37 ........................... & 83.81955700 & -5.31254300 & 846.89 & 1300.70 & 780.42 & 29.92 & 29.71 & 30.14 & 30.40 & 31.00 & 31.21 \\
\hline
\end{tabular}

NotE.-A description of the columns is given in $\S 5.1$. 
TABLE 8

X-Ray Parameters of the Class II Candidates

\begin{tabular}{|c|c|c|c|c|c|c|c|c|}
\hline Sequence Number & COUP ID & $\begin{array}{l}\log N_{\mathrm{H}} \\
\left(\mathrm{cm}^{-2}\right)\end{array}$ & $\begin{array}{c}k T_{1} \\
(\mathrm{keV})\end{array}$ & $\begin{array}{c}\log \mathrm{EM}_{1} \\
\left(\mathrm{~cm}^{-3}\right)\end{array}$ & $\begin{array}{c}k T_{2} \\
(\mathrm{keV})\end{array}$ & $\begin{array}{c}\log \mathrm{EM}_{2} \\
\left(\mathrm{~cm}^{-3}\right)\end{array}$ & $\begin{array}{c}\log L_{h, c} \\
\left(\operatorname{ergs~s}^{-1}\right)\end{array}$ & $\begin{array}{c}\log L_{t, c} \\
\left(\operatorname{ergs~s}^{-1}\right)\end{array}$ \\
\hline 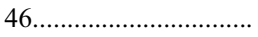 & 12 & $21.33 \pm 0.18$ & $14.34 \pm 11.68$ & $52.44 \pm 0.05$ & $\ldots$ & . & 29.48 & 29.64 \\
\hline 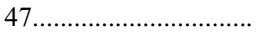 & 13 & $20.00 \pm 2.12$ & $12.58 \pm 15.00$ & $51.51 \pm 0.17$ & $\ldots$ & $\ldots$ & 28.54 & 28.71 \\
\hline $48 \ldots \ldots \ldots \ldots \ldots \ldots \ldots \ldots \ldots \ldots \ldots \ldots \ldots \ldots \ldots$ & 21 & $20.79 \pm 2.06$ & $0.68 \pm 0.11$ & $51.63 \pm 0.17$ & $1.77 \pm 0.47$ & $51.89 \pm 0.12$ & 28.38 & 29.04 \\
\hline 49 & 29 & $21.59 \pm 0.03$ & $0.81 \pm 0.06$ & $52.56 \pm 0.13$ & $3.13 \pm 0.32$ & $52.82 \pm 0.03$ & 29.59 & 30.04 \\
\hline $52 \ldots \ldots \ldots \ldots \ldots \ldots \ldots \ldots \ldots \ldots \ldots \ldots \ldots$ & 37 & $20.00 \pm 2.34$ & $0.67 \pm 0.33$ & $51.13 \pm 0.63$ & $\ldots$ & $\ldots$ & 26.70 & 28.10 \\
\hline 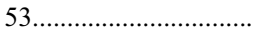 & 40 & $20.35 \pm 2.00$ & $0.86 \pm 0.11$ & $51.55 \pm 0.15$ & $6.29 \pm 4.63$ & $51.64 \pm 0.08$ & 28.62 & 28.99 \\
\hline 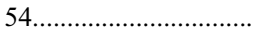 & 44 & $21.79 \pm 0.32$ & $1.14 \pm 0.35$ & $51.50 \pm 0.29$ & $\ldots$ & $\ldots$ & 27.64 & 28.44 \\
\hline
\end{tabular}

Notes.-Table 8 is published in its entirety in the electronic edition of the Astrophysical Journal. A portion is shown here for guidance regarding its form and content.

TABLE 9

X-Ray Parameters of the Class III Candidates

\begin{tabular}{|c|c|c|c|c|c|c|c|c|}
\hline Sequence Number & COUP ID & $\begin{array}{l}\log N_{\mathrm{H}} \\
\left(\mathrm{cm}^{-2}\right)\end{array}$ & $\begin{array}{c}k T_{1} \\
(\mathrm{keV})\end{array}$ & $\begin{array}{c}\log \mathrm{EM}_{1} \\
\left(\mathrm{~cm}^{-3}\right)\end{array}$ & $\begin{array}{c}k T_{2} \\
(\mathrm{keV})\end{array}$ & $\begin{array}{c}\log \mathrm{EM}_{2} \\
\left(\mathrm{~cm}^{-3}\right)\end{array}$ & $\begin{array}{c}\log L_{h, c} \\
\left(\operatorname{ergs~s}^{-1}\right)\end{array}$ & $\begin{array}{c}\log L_{t, c} \\
\left(\operatorname{ergs~s}{ }^{-1}\right)\end{array}$ \\
\hline 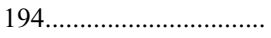 & 1 & $20.67 \pm 0.43$ & $0.85 \pm 0.12$ & $52.17 \pm 0.11$ & $4.37 \pm 0.95$ & $52.62 \pm 0.04$ & 29.50 & 29.84 \\
\hline 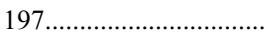 & 6 & $21.12 \pm 0.12$ & $0.78 \pm 0.09$ & $52.16 \pm 0.12$ & $2.32 \pm 0.27$ & $52.68 \pm 0.04$ & 29.32 & 29.79 \\
\hline 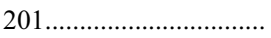 & 7 & $20.93 \pm 0.04$ & $0.83 \pm 0.01$ & $53.58 \pm 0.02$ & $2.30 \pm 0.08$ & $53.94 \pm 0.01$ & 30.57 & 31.08 \\
\hline 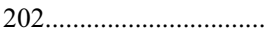 & 9 & $21.37 \pm 0.03$ & $0.83 \pm 0.02$ & $53.59 \pm 0.03$ & $3.04 \pm 0.11$ & $53.87 \pm 0.01$ & 30.64 & 31.08 \\
\hline 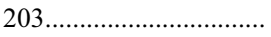 & 10 & $20.00 \pm 1.21$ & $0.74 \pm 0.13$ & $51.42 \pm 0.21$ & $\ldots$ & $\ldots$ & 27.11 & 28.39 \\
\hline 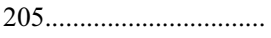 & 14 & $20.81 \pm 0.14$ & $0.69 \pm 6.24$ & $51.64 \pm 1.00$ & $9.23 \pm 15.00$ & $51.19 \pm 0.21$ & 28.25 & 28.82 \\
\hline
\end{tabular}

Notes.-Table 9 is published in its entirety in the electronic edition of the Astrophysical Journal. A portion is shown here for guidance regarding its form and content.

TABLE 10

Upper Limits of the X-Ray-undetected Class II and III Candidates

\begin{tabular}{|c|c|c|c|c|c|c|c|c|c|c|c|c|c|}
\hline \multirow{2}{*}{$\begin{array}{c}\text { SEQUENCE } \\
\text { Number }\end{array}$} & \multirow{2}{*}{$\begin{array}{c}\text { ID } \\
\text { H97 }\end{array}$} & \multirow{2}{*}{$\begin{array}{c}\text { R.A. } \\
\text { (J2000.0) } \\
(\mathrm{deg})\end{array}$} & \multirow{2}{*}{$\begin{array}{c}\text { DeCL. } \\
(\mathrm{J} 2000.0) \\
(\mathrm{deg})\end{array}$} & \multicolumn{2}{|c|}{ Counts } & \multirow{2}{*}{$\begin{array}{c}\text { EXPOSURE } \\
(\mathrm{ks})\end{array}$} & \multirow{2}{*}{$\begin{array}{c}\log L_{h, c}^{1} \\
\left(\operatorname{ergs~s}^{-1}\right)\end{array}$} & \multirow{2}{*}{$\begin{array}{c}\log L_{h, c}^{2} \\
\left(\operatorname{ergs~s}^{-1}\right)\end{array}$} & \multirow{2}{*}{$\begin{array}{c}\log L_{h, c}^{3} \\
\left(\operatorname{ergs~s}^{-1}\right)\end{array}$} & \multirow{2}{*}{$\begin{array}{c}\log L_{t, c}^{1} \\
\left(\operatorname{ergs~s}^{-1}\right)\end{array}$} & \multirow{2}{*}{$\begin{array}{c}\log L_{t, c}^{2} \\
\left(\operatorname{ergs~s}^{-1}\right)\end{array}$} & \multirow{2}{*}{$\begin{array}{c}\log L_{t, c}^{3} \\
\left(\operatorname{ergs~s}^{-1}\right)\end{array}$} & \multirow[b]{2}{*}{ Class } \\
\hline & & & & Hard & Total & & & & & & & & \\
\hline $51 \ldots$ & 85 & 83.69498179 & -5.35801543 & 117 & 130 & 0.76 & 28.83 & 27.89 & 29.10 & 28.73 & 28.54 & 29.38 & II \\
\hline $55 \ldots \ldots \ldots \ldots \ldots$ & 108 & 83.70820425 & -5.31230548 & 83 & 66 & 0.45 & 28.92 & 27.99 & 29.19 & 28.66 & 28.47 & 29.31 & II \\
\hline $66 \ldots \ldots \ldots \ldots \ldots$ & 179 & 83.74109068 & -5.47828377 & 79 & 237 & 0.73 & 28.67 & 27.73 & 28.94 & 29.00 & 28.81 & 29.66 & II \\
\hline $74 \ldots \ldots \ldots \ldots \ldots . . . . . .$. & 219 & 83.75862700 & -5.30628963 & 27 & 35 & 0.77 & 28.19 & 27.26 & 28.46 & 28.15 & 27.96 & 28.81 & II \\
\hline $98 \ldots \ldots \ldots \ldots \ldots \ldots$ & 294 & 83.77694276 & -5.51927167 & 32 & 39 & 0.71 & 28.29 & 27.35 & 28.56 & 28.23 & 28.04 & 28.89 & II \\
\hline $113 \ldots \ldots \ldots \ldots \ldots$ & 402 & 83.80437902 & -5.56763586 & 57 & 66 & 0.66 & 28.58 & 27.64 & 28.85 & 28.50 & 28.31 & 29.15 & II \\
\hline $123 \ldots \ldots \ldots \ldots$ & 449 & 83.81012490 & -5.55521463 & 51 & 500 & 0.68 & 28.51 & 27.57 & 28.78 & 29.36 & 29.17 & 30.01 & II \\
\hline $153 \ldots \ldots \ldots \ldots \ldots$ & 682 & 83.83838625 & -5.55481843 & 47 & 57 & 0.71 & 28.46 & 27.52 & 28.73 & 28.40 & 28.21 & 29.05 & II \\
\hline $166 \ldots \ldots \ldots \ldots \ldots \ldots$ & 789 & 83.85709585 & -5.49312187 & 24 & 29 & 0.76 & 28.14 & 27.20 & 28.41 & 28.08 & 27.89 & 28.73 & II \\
\hline $339 \ldots \ldots \ldots \ldots \ldots$ & 729 & 83.84592814 & -5.49483049 & 23.07 & 28.00 & 0.72 & 28.12 & 27.21 & 28.41 & 27.92 & 27.85 & 28.77 & III \\
\hline $210 \ldots \ldots \ldots \ldots \ldots$ & 62 & 83.67848933 & -5.42114559 & 38.07 & 55.00 & 0.75 & 28.31 & 27.40 & 28.60 & 28.19 & 28.12 & 29.04 & III \\
\hline
\end{tabular}



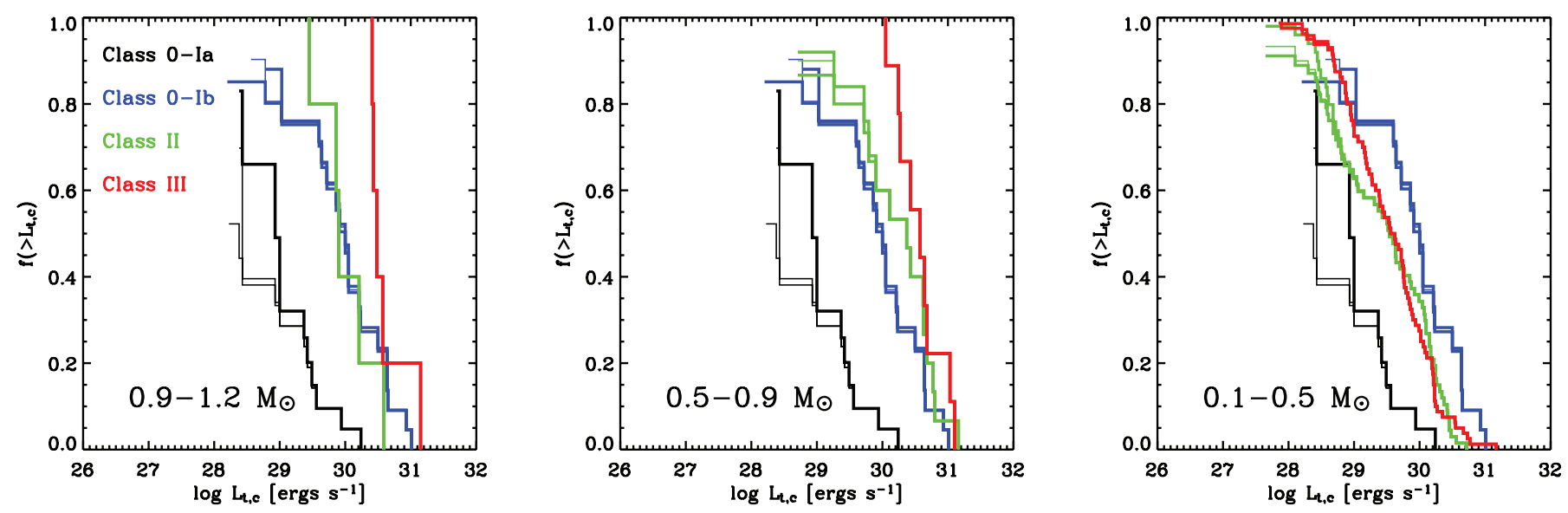

FIG. 8.-Maximum likelihood cumulative XLF in the total $(0.2-8.0 \mathrm{keV})$ energy band of the four NIR classes. The three panels refer to Class II and III stars in three

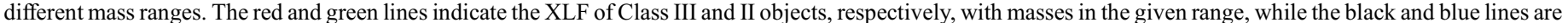

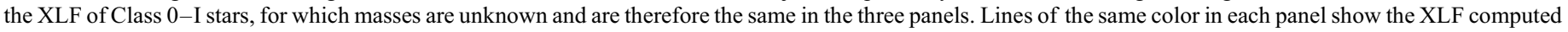

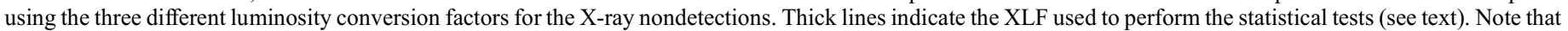
the distributions do not reach $f=100 \%$ because of the presence of upper limits.

estimator. For Class II and III sources, we considered separately stars in the three mass ranges used by Preibisch \& Feigelson (2005). Figure 8 shows the comparison of the XLFs of the four populations. The red and green lines indicate the XLF of Class III and II objects, respectively, with masses in the given range, while the black and blue lines are the XLFs of Class $0-\mathrm{Ia}$ and $0-\mathrm{Ib}$ stars, for which masses are unknown and are therefore the same in the three panels. Lines of the same color in each panel show the XLF obtained using the three different count rate-to-luminosity conversion factors adopted for the X-ray nondetections of each subsample and give an idea of the uncertainty of the XLFs. We removed from this analysis the four objects of our sample of Class $0-$ Ia protostars without detections at $4.5 \mu \mathrm{m}$. Since this band is one of the most sensitive bands and the least contaminated by nebulosity, these objects could be PAH-contaminated sources or even knots of nebulosity. By discarding these objects, we are more confident to consider a sample of protostar candidates as less contaminated as possible. The figure shows that for the two highest mass ranges, the X-ray luminosities of Class III stars are highly elevated with respect to those of Class II stars and Class $0-$ Ib objects. The latter two classes have very similar XLFs and are significantly brighter than Class 0 -Ia stars.

Because the Class $0-\mathrm{Ia}$ and $0-\mathrm{Ib}$ stars in our sample are heavily absorbed, a note of caution is needed. Since absorption precludes the observation of the soft part of the intrinsic source $\mathrm{X}$-ray spectra, we cannot rule out the presence of soft, completely absorbed emission from Class $0-$ I stars. Nevertheless, we know from previous studies of CTTSs that a very soft component, $k T<0.3 \mathrm{keV}$, in the few cases in which it is observed, is mainly due to accretion (Kastner et al. 2002; Schmitt et al. 2005; Güdel et al. 2007; Argiroffi et al. 2007; Huenemoerder et al. 2007; Grosso et al. 2007), while harder components are due to coronal-like emission. This is in agreement with the previous finding for Class II stars in other regions (e.g., Stelzer \& Schmitt 2004), where the X-ray spectra of Class II stars were found to include an additional softer emission component compared to WTTSs. Our Orion stars are too heavily absorbed to detect this soft component.

Coronal emission, as analyzed with resolution spectra from charge-coupled devices, can usually be decomposed into softer $(\sim 0.8-1.0 \mathrm{keV})$ and harder $(\sim 2 \mathrm{keV})$ components (Preibisch et al. 2005). The soft coronal component, like the accretion com- ponent, might remain unobserved in heavily extincted sources such as our Class 0-I stars, and thus the X-ray luminosities in the total band can underestimate the true emission in such objects. In addition, magnetic flare components can be strong around $0.5-2.0 \mathrm{keV}$ and dominate the X-ray flux. For these reasons, we also considered the comparison of the XLFs of different classes, by restricting the analysis to the X-ray luminosities in the hard (2.0-8.0 keV) energy band. We note that we recomputed with PWDetect the upper limits in the hard band for a total of 31 undetected objects, assuming a threshold of $4.8 \sigma$ estimated from the background in this band. Among these objects, 12 are flagged by the PWDetect code as affected by an X-ray-detected source nearby, which therefore raises the upper limit count rate. In 2 cases out of 12 , however, the asymmetry of the Chandra PSF at large off-axis angles is such as to make PWDetect clearly overestimate the contribution of the detected source to the computed upper limit at the desired position, which is instead dominated by the background; in these instances we computed the upper limit from the background alone. The results of this analysis are shown in Figure 9, analogous to Figure 8 but for the hard energy band.

To check if these results are statistically significant, we performed statistical tests developed for "survival analysis," suited for the analysis of censored data sets. The probabilities that the $\mathrm{X}$-ray luminosities of the stars in two samples are drawn from the same parent distribution were computed with the ASURV ${ }^{11}$ package (Feigelson \& Nelson 1985). The results of this analysis are given in Tables 11 and 12, where, for each pair of samples, we list the probabilities computed using the five tests of the ASURV package. We note that the tests were performed in the most conservative way, i.e., by considering for each pair of samples the nearest XLFs among those computed with different conversion factors for the upper limits.

The tests show that Class 0 -Ia and $0-$ Ib stars have statistically different XLFs in both the hard and total bands, at a significance level larger than 99\%; Class 0 -Ia and II are different at a significance level of $99 \%$ for the $0.9-1.2$ and $0.5-0.9 M_{\odot}$ mass ranges. The difference is marginal, in both the total and hard bands, for the $0.1-0.5 M_{\odot}$ mass range. The difference between Class II and III objects is quite marginal (between $85 \%$ and $95 \%$ )

\footnotetext{
${ }^{11}$ Astronomy Survival Analysis available from the StatCodes http://www astro.psu.edu/statcodes.
} 

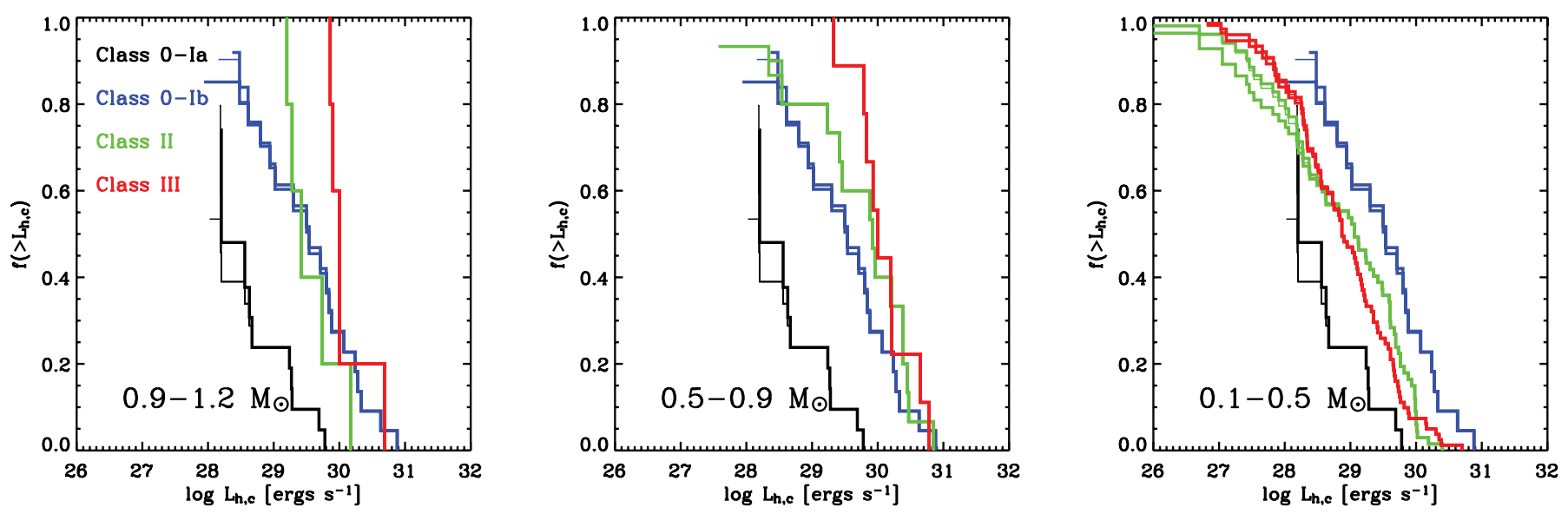

FIG. 9. - Maximum likelihood cumulative XLF in the hard (2.0-8.0 keV) energy band of the four NIR classes. The figure is analogous to Fig. 8.

TABLE 11

Probabilities of Correlation between XLFs in the Total Band

\begin{tabular}{|c|c|c|c|c|c|c|c|}
\hline $\begin{array}{l}\text { Mass } \\
\left(M_{\odot}\right)\end{array}$ & $\begin{array}{c}P_{1} \\
(\%)\end{array}$ & $\begin{array}{c}P_{2} \\
(\%)\end{array}$ & $\begin{array}{c}P_{3} \\
(\%)\end{array}$ & $\begin{array}{c}P_{4} \\
(\%)\end{array}$ & $\begin{array}{c}P_{5} \\
(\%)\end{array}$ & Sample 1 & Sample 2 \\
\hline & 0.23 & 0.21 & 3.13 & 0.36 & 0.25 & Class 0-Ia & Class $0-\mathrm{Ib}$ \\
\hline \multirow[t]{3}{*}{$0.9-1.2 \ldots \ldots \ldots \ldots \ldots \ldots \ldots \ldots$} & 0.35 & 0.07 & 0.03 & 0.46 & 0.45 & Class 0-Ia & Class II \\
\hline & 94.75 & 94.99 & 62.55 & 97.88 & 97.73 & Class $0-\mathrm{Ib}$ & Class II \\
\hline & 6.79 & 5.10 & 16.15 & 16.15 & $\ldots$ & Class II & Class III \\
\hline \multirow[t]{3}{*}{$0.5-0.9 \ldots \ldots \ldots \ldots \ldots \ldots \ldots \ldots$} & 0.04 & 0.02 & 0.06 & 0.05 & 0.03 & Class 0-Ia & Class II \\
\hline & 35.94 & 35.60 & 58.58 & 36.77 & 36.64 & Class 0-Ib & Class II \\
\hline & 33.13 & 32.21 & 14.63 & 31.51 & 32.68 & Class II & Class III \\
\hline \multirow[t]{3}{*}{$0.1-0.5$} & 5.28 & 5.64 & 23.77 & 6.04 & 5.53 & Class 0-Ia & Class II \\
\hline & 3.41 & 2.02 & 1.52 & 3.43 & 2.88 & Class 0-Ib & Class II \\
\hline & 86.08 & 86.09 & 40.72 & 80.37 & 80.34 & Class II & Class III \\
\hline
\end{tabular}

Notes.-Probabilities of correlation between the XLFs in the total band $(0.2-8.0 \mathrm{keV})$ of two samples, computed with the ASURV package (see $\S 5.2$ ). $P_{1}$ and $P_{2}$ are the probabilities computed using the Gehan generalized Wilcoxon test with permutation and hypergeometric variance, respectively, $P_{3}$ is the probability from the Logrank test, while $P_{4}$ and $P_{5}$ are the probabilities from the Peto and Peto and from the Peto and Prentice generalized Wilcoxon tests, respectively.

TABLE 12

Probabilities of Correlation between the XlFs in the Hard Band

\begin{tabular}{crrrrrll}
\hline \hline $\begin{array}{c}\text { Mass } \\
\left(M_{\odot}\right)\end{array}$ & $\begin{array}{c}P_{1} \\
(\%)\end{array}$ & \multicolumn{1}{c}{$\begin{array}{c}P_{2} \\
(\%)\end{array}$} & $\begin{array}{c}P_{3} \\
(\%)\end{array}$ & \multicolumn{1}{c}{$\begin{array}{c}P_{4} \\
(\%)\end{array}$} & $\begin{array}{c}P_{5} \\
(\%)\end{array}$ & Sample 1 & Sample 2 \\
\hline & 0.36 & 0.36 & 2.99 & 0.53 & 0.47 & Class 0-Ia & Class 0-Ib \\
& 0.57 & 0.15 & 0.08 & 0.72 & 0.73 & Class 0-Ia & Class II \\
& 94.84 & 95.04 & 46.15 & 93.70 & 94.39 & Class 0-Ib & Class II \\
& 6.54 & 4.77 & 9.37 & 9.37 & $\ldots$ & Class II & Class III \\
& 0.08 & 0.05 & 0.89 & 0.12 & 0.08 & Class 0-Ia & Class II \\
& 28.18 & 27.57 & 56.83 & 29.37 & 28.97 & Class 0-Ib & Class II \\
& 62.80 & 62.59 & 34.13 & 61.58 & 62.47 & Class II & Class III \\
& 14.23 & 14.57 & 45.40 & 16.79 & 16.58 & Class 0-Ia & Class II \\
& 3.62 & 2.19 & 2.05 & 3.70 & 3.02 & Class 0-Ib & Class II \\
& 46.63 & 46.63 & 91.93 & 50.19 & 50.23 & Class II & Class III \\
\hline
\end{tabular}

Notes.-Probabilities of correlation between the XLFs in the hard band (2.0-8.0 keV) of two samples, computed with the ASURV package (see $\S 5.2$ ). The probabilities are those described in Table 11. 
for the highest mass range, while for lower mass stars, Class II and III stars do not show any significant difference.

These results clearly indicate an evolution of the X-ray activity from Class 0-Ia, the least X-ray-luminous objects, up to Class III, which show the highest X-ray luminosities, at least for stars with mass larger than $0.9 M_{\odot}$. Although the difference in the intrinsic X-ray activity between Class II and III stars is quite marginal, it is in agreement with previous results indicating that the X-ray luminosities of accretors in the ONC are smaller than those of nonaccretors (Flaccomio et al. 2003c; Preibisch et al. 2005). Note, however, that these results cannot be compared directly because of the different criteria adopted to select the two populations.

The most significant and novel result is the difference in X-ray activity level between Class 0 -Ia and II and between Class $0-$ Ia and $0-\mathrm{Ib}$ sources. Our results are even more robust if we include the four objects without detections at $4.5 \mu \mathrm{m}$.

Based on our selection criterion, Class 0 -Ia objects are protostars with SEDs increasing up to $8.0 \mu \mathrm{m}$, while Class 0 -Ib objects are protostars with SEDs increasing up to $4.5 \mu \mathrm{m}$ and decreasing at longer wavelengths. Figure $10 a$ shows the absorption-corrected X-ray luminosities plotted versus the [5.8] - [8.0] color, which discriminates Class 0-Ia stars (squares) from Class 0-Ib ones (filled circles), the former being redder than $[5.8]-[8.0] \simeq 1.1$ and the latter bluer than this value. This plot indicates a trend of increasing $\mathrm{X}$-ray luminosity with decreasing $[5.8]-[8.0]$ color. Figure $10 b$ shows the same luminosities as a function of the [3.6] - [4.5] color. While the latter color does not discriminate between Class $0-$ Ia and $0-\mathrm{Ib}$ objects, it clearly confirms that, from the X-ray point of view, Class $0-$ Ia and $0-\mathrm{Ib}$ objects belong to two different populations. This again suggests to us that the contamination by reddened Class II objects can be very high for Class $0-$ Ib objects, while the Class 0 -Ia sample is dominated by true protostars. We could then be witnessing the onset of X-ray activity in very young YSOs.

Class $0-$ I objects are affected by larger absorptions with respect to the other classes, as we confirm in $\S 5.3$ using the X-rayderived $N_{\mathrm{H}}$ values. Can this fact result in a systematic underestimation of $L_{\mathrm{X}}$ values for Class 0 -I stars? Although we do apply absorption corrections to the observed X-ray fluxes when deriving luminosities in both the $0.5-8.0$ and $2.0-8.0 \mathrm{keV}$ bands, these are corrections to the observed X-ray spectra and emission from relatively cool plasma would remain unobserved, and therefore unaccounted for, in heavily observed sources.

In order to verify that the X-ray luminosities of heavily absorbed Class $0-$ I objects are accurately corrected for absorption, we assumed that these sources have X-ray spectra similar to Class II and III objects and computed through extensive simulations the effect of varying the absorbing hydrogen column density on the determination of X-ray luminosities.

For this purpose we considered the best-fit spectra of a subsample of $\sim 200$ Class II and III objects with small absorption $\left(\log N_{\mathrm{H}}<21.5 \mathrm{~cm}^{-2}\right)$, consisting of either one or two thermal components. For each of these spectra, using XPSEC and the fakeit command, we computed 10 simulated spectra for each of five different $N_{\mathrm{H}}$ values in the range $10^{21} \mathrm{~cm}^{-2}<N_{\mathrm{H}}<$ $10^{23} \mathrm{~cm}^{-2}$. We then analyzed the simulated spectra with a procedure analogous to that used by Getman et al. (2005a) for the analysis of the observed spectra. Each spectrum was rebinned so as to have a minimum of 15 photons per channel and was then fitted with both an isothermal and a two-component model. After adopting the simplest of the two models that gave a statistically acceptable fit, we computed the unabsorbed flux in the 0.5 8.0 and $2.0-8.0 \mathrm{keV}$ bands. We then computed the absorption-
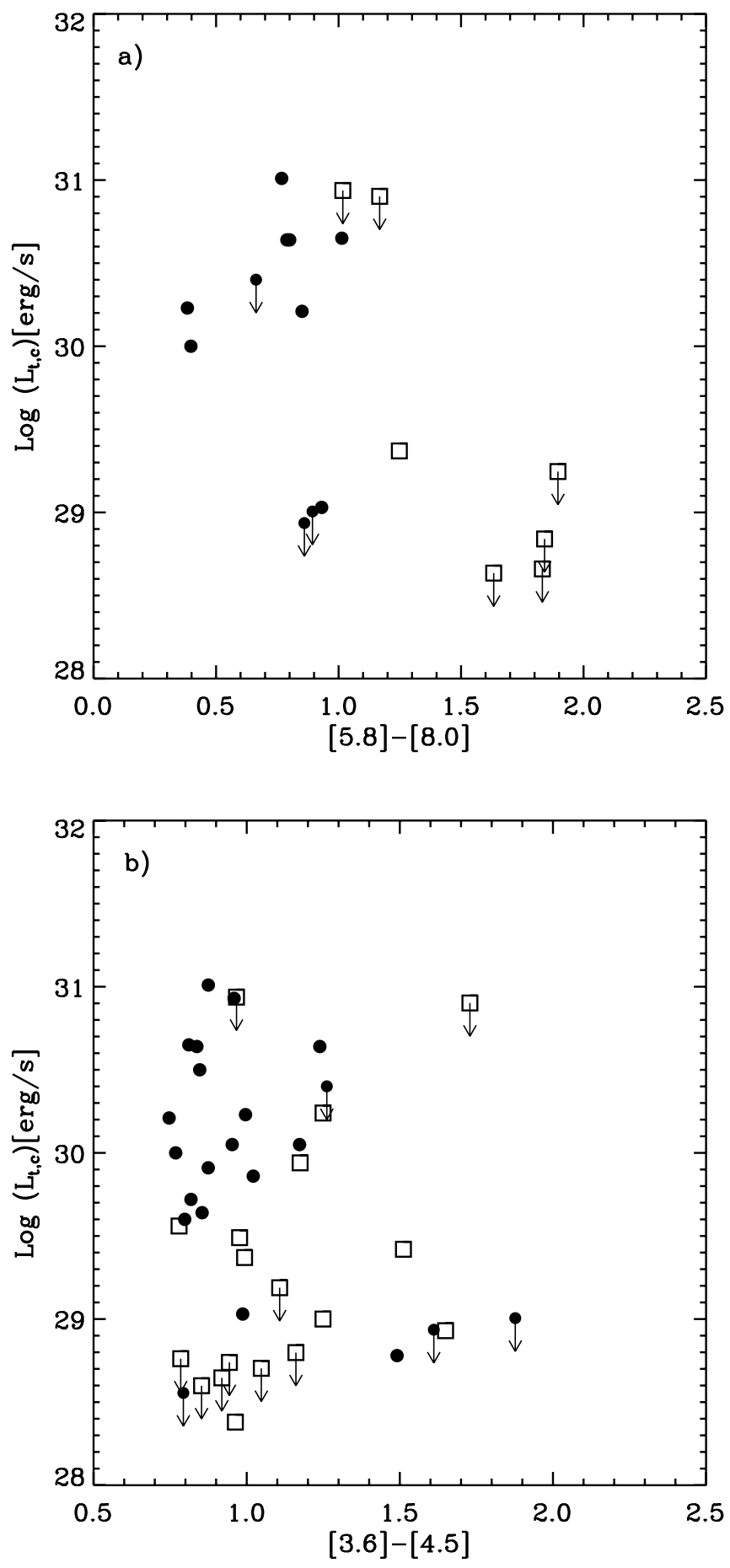

FIG. 10.-Absorption-corrected X-ray luminosity of Class 0-Ia stars (squares) and Class 0-Ib stars (circles) as a function of the $(a)[5.8]-[8.0]$ and $(b)[3.6]-$ [4.5] colors. Upper limits are indicated by arrows.

corrected XLFs for each of the five absorption values. Most of the simulated sources did not have enough photons when absorbed by $N_{\mathrm{H}}=10^{23} \mathrm{~cm}^{-2}$. In order to avoid selection effects, since we are not accounting for upper limits, we could only compare the XLFs for the 115 sources whose spectra were bright enough to be analyzed even when considering the highest absorption value. The result is that the five XLFs for the $0.5-8.0 \mathrm{keV}$ luminosities have medians within $\sim 0.1$ dex but tend to become wider as the $N_{\mathrm{H}}$ is increased. The five XLFs for $2.0-8.0 \mathrm{keV}$ luminosities are instead very similar in shape and with medians within 0.03 dex, except for the one relative to the highest absorption values, 
which is brighter by $\sim 0.1 \mathrm{dex}$. We conclude that, in the assumption that Class $0-$ I sources have the same spectra of Class II-III ones, the X-ray luminosities we derive are not underestimated.

We cannot exclude that some of the Class $0-\mathrm{Ib}$ stars are actually heavily absorbed Class II stars (see Fig. 3). However, even in this case, our result would plausibly indicate a significant evolution of X-ray activity in the early stages of YSO formation.

Due to various IR sensitivity limitations at very high obscurations, the Class 0-I sampling might be restricted to higher masses (with stronger IR bolometric luminosities) and, through the $L_{\mathrm{X}^{-}}$ mass correlation, would have higher $L_{\mathrm{X}}$ values introducing a bias. But we find that these heavily obscured stars have lower, not higher, $L_{\mathrm{X}}$ values, further supporting our result.

Another bias may be introduced by the requirement that all stars considered have IRAC detections in one or more bands. Therefore, it is possible that lower mass (i.e., bolometrically weaker) Class III stars have been systematically omitted from the sample, leaving behind too many higher mass, i.e., stronger $L_{\mathrm{X}}$, stars. But this is not relevant as the XLF comparisons in Figures 8 and 9 are binned by mass. We might have few $0.1-0.5 M_{\odot}$ Class III stars, but those shown should be unbiased in $L_{\mathrm{X}}$.

Another possible worry is that flares could dominate the flux from some of our objects, and our X-ray spectra could pertain to the flaring state rather than the quiescent state. We have verified that this is not the case by using the technique described in Wolk et al. (2005) to recompute X-ray spectra and fluxes for our stars using photons detected only during nonflaring intervals. Fits to these new spectra yield XLFs that do not differ significantly from those shown in Figure 8. We have also verified that, using these new spectra, the results discussed in the next section do not change significantly.

We note that our results are consistent with the nondetection of Class 0 sources of Serpens (Giardino et al. 2007). In fact, their limiting sensitivity $\left(4.0 \times 10^{29} \mathrm{ergs} \mathrm{s}^{-1}\right.$ in the hard band), estimated assuming temperature and absorption similar to those we found for our X-ray-detected Class 0 -I sources, corresponds to the highest 10th percentile of the XLF of our Class 0-Ia stars. Considering the small size of the Serpens Class 0 star sample, their nondetection is not surprising.

\subsection{Gas and Dust Absorption}

Figure 11 shows the Spitzer IRAC [3.6] - [4.5] color versus Chandra X-ray median energy of all selected sources. The typical uncertainty in the median energy has been estimated using the results of the MARX simulations made in Getman et al. (2006), where the median energy errors were computed as a function of the source net counts. We used the value of $0.3 \mathrm{keV}$, corresponding to objects with $\operatorname{Med} E \gtrsim 2 \mathrm{keV}$ and with about 30 net counts, which is the minimum of net counts we have for Class $0-\mathrm{I}$ objects. The typical uncertainty in the IRAC color has been estimated assuming a 5\% uncertainty for the IRAC magnitudes.

As discussed in Getman et al. (2007), high X-ray median en$\operatorname{ergy}(\mathrm{Med} E \gtrsim 1.7 \mathrm{keV})$ can be considered as an indicator of absorbing column density that arises from gas, primarily metallic elements (e.g., N, O, Ne, Mg, Si, Ar, Fe). On the other hand, if the object is known to be a protostar, the [3.6] - [4.5] color is primarily a measure of the density of grains in the local infalling envelope. Thus, the correlation between these two quantities, first found in Getman et al. (2007), has been interpreted by these authors as evidence of absorption from the protostellar envelope rather than from the ambient molecular cloud material. Although our data do not cover the whole X-ray median energy, they show a trend consistent with that found using the IC $1396 \mathrm{~N}$ data, also shown in Figure 11. Nevertheless, the interpretation of our data is

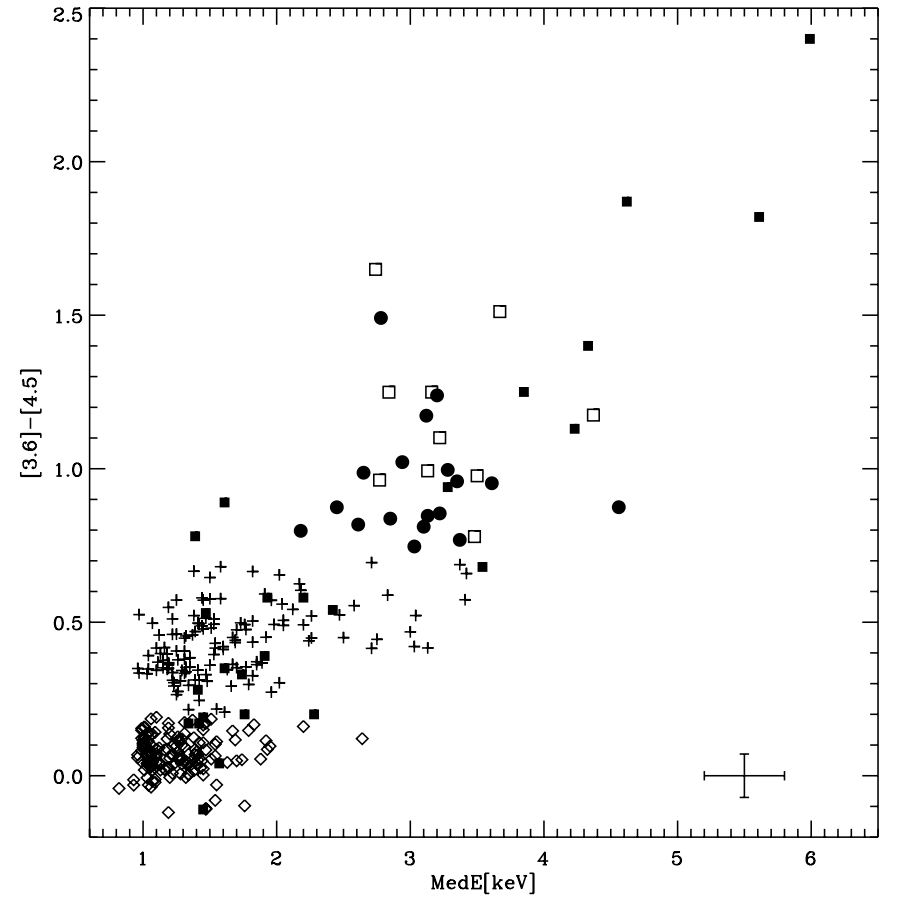

FIG. 11.-Spitzer IRAC [3.6] - [4.5] color vs. Chandra X-ray median energy of all selected sources. The different classes of ONC objects are indicated using the same symbols as in Fig. 5, while filled squares are the values of the YSO in IC $1396 \mathrm{~N}$ taken from Getman et al. (2007). Typical error bars in the median energy and in the IRAC colors are also shown.

not as clear as it was in IC $1396 \mathrm{~N}$, since the ONC background is quite complex while the maximum line-of-sight column is $A_{V} \sim 10$ mag.

Figure 12 compares the absorbing column density $N_{\mathrm{H}}$ for the different populations. In the left panel, $\log N_{\mathrm{H}}$ is plotted for the $\mathrm{X}$-ray-detected objects as a function of the unabsorbed X-ray luminosity $L_{t, c}$. The right panel shows, for each class, the median values of the absorbing column density distributions, values between the first and third quartiles, and values between the minimum and the maximum of the distributions of the $\log N_{\mathrm{H}}$ values. Note that only objects with errors in $\log N_{\mathrm{H}}$ smaller than 0.5 have been considered. However, the median values of the distribution do not change significantly if all data are considered.

A clear trend of increasing absorbing column density from Class III to Class $0-$ I can be seen in both the left and right panels. This trend is expected since Class 0-I objects are characterized by a large amount of infalling material and are also typically embedded in the molecular cloud; a smaller amount of obscuring material is instead present for Class II objects that have shed their envelope and formed a circumstellar disk, while the extinction suffered from Class III objects is due to interstellar material only (both within the cloud and on the line of sight to the cloud).

We note that the estimate of the $N_{\mathrm{H}}$ values of Class $0-\mathrm{I}$ stars is based primarily on the hard component of the X-ray spectrum. That is, at the temperatures of the protostars $\left(k T_{\mathrm{av}} \sim 2 \mathrm{keV}\right)$ and with $\log N_{\mathrm{H}} \sim 23 \mathrm{~cm}^{-2}$, the observed flux is almost entirely due to the hard component of the X-ray spectrum. At the same temperatures, if the $N_{\mathrm{H}}$ was smaller, the total flux would be distributed between the soft and hard component and the shape of the spectrum would be different. Our technique should thus be the most accurate for objects with high $N_{\mathrm{H}}$ values, thus yielding particularly good $N_{\mathrm{H}}$ values for the, on average, heavily embedded protostars. Therefore, we argue that the low XLFs for protostars we have derived (see Fig. 9) are unlikely to be due to errors in our 

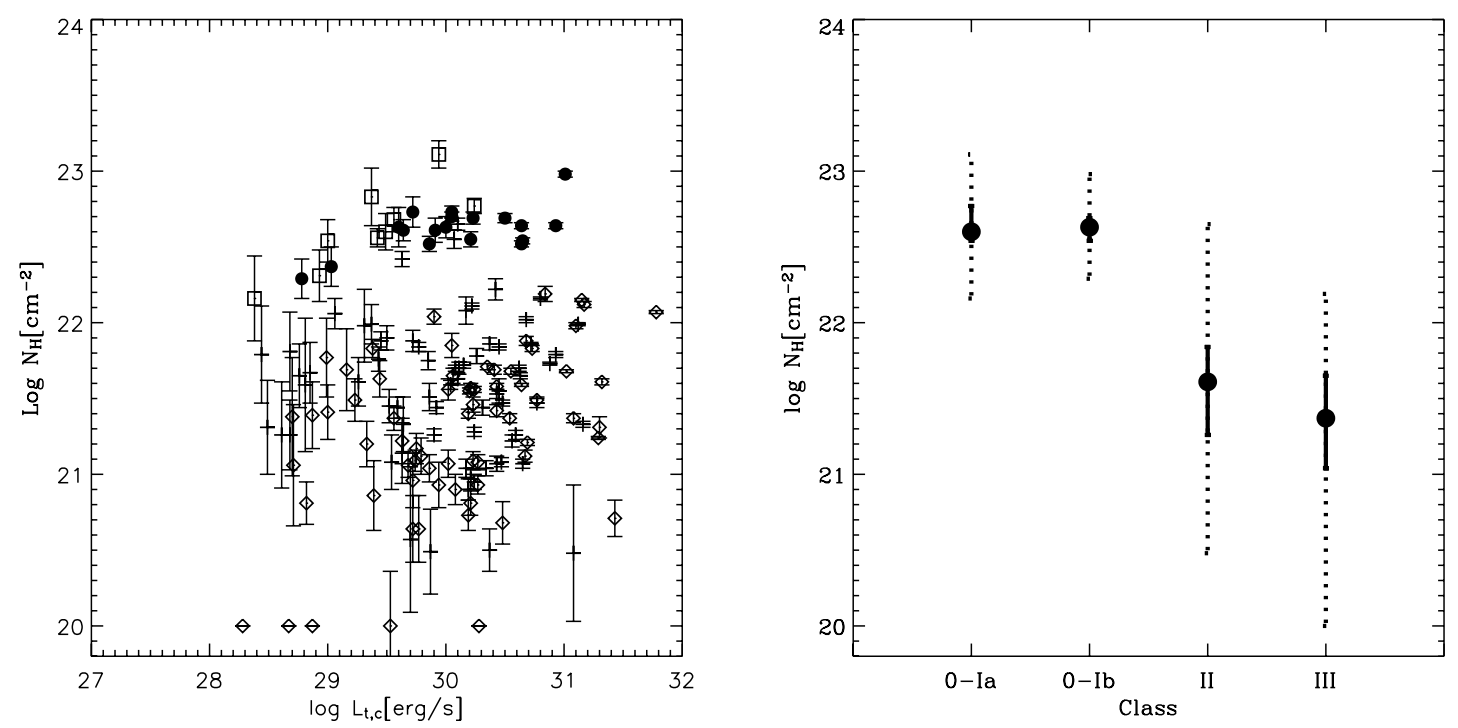

Fig. 12. - Left: $\log N_{\mathrm{H}}$ values as a function of the unabsorbed X-ray luminosity $L_{t, c}$ for the X-ray-detected objects. Symbols are as in Fig. 5. Right: For each class, the median values of the absorbing column density distributions are indicated by the big black filled circles. Solid bars indicate the values of log $N_{\mathrm{H}}$ included between the first and third quartiles, while the dashed lines (partially hidden by solid bars) indicate the values included between the minimum and the maximum of the distributions of the $\log N_{\mathrm{H}}$ values. Note that only objects with errors in $\log N_{\mathrm{H}}$ smaller than 0.5 were considered.

estimates of the X-ray absorption toward these objects, as confirmed from the analysis of the hard XLFs and the simulated spectra of Class II and III stars (see $\S 5.2$ ).

\subsection{Evolution of YSO X-Ray Spectra and Temporal Characteristics}

Figure 13 shows the results of the comparison of the average plasma temperature $k T_{\text {av }}$ among the different classes. For X-ray spectra fitted with two isothermal components, $k T_{\text {av }}$ was computed as the mean of the soft and hard $k T$ spectral components, weighted by their respective emission measures; for spectra fitted with a single component $k T_{\mathrm{av}}=k T$. The $k T_{\mathrm{av}}$ values for the $\mathrm{X}$-ray-detected objects of the different populations are plotted as a function of the unabsorbed X-ray luminosity $L_{t, c}$. Note that only objects with errors on $k T_{\text {av }}$ smaller than $1 \mathrm{keV}$ have been considered. However, the distributions do not change significantly if all data are considered. The bottom right panel of Figure 13 shows, for each class, the median values of the average plasma temperatures, values between the first and third quartiles, and values between the minimum and the maximum of the distributions of the $k T_{\mathrm{av}}$ values. No trend in plasma energy is found as a function of evolutionary stage from Class 0 -Ia to Class III systems.

A trend of increasing average plasma temperature with increasing unabsorbed X-ray luminosity is clearly evident for all the samples, as has often been reported for other types of coronal sources (e.g., Schmitt et al. 1990). A similar result is obtained by considering only the subsample of stars with X-ray spectra fitted with two temperature components: high-luminosity stars tend to have high values of the ratio between the high- and lowtemperature emission measures $\left(\mathrm{EM}_{2} / \mathrm{EM}_{1} \sim 2.5\right)$, suggesting that the hot plasma component dominates over the cold one for the high-luminosity stars. No significant difference between the classes is observed in Figure 13.

Finally, we checked if ONC stars of different classes show different X-ray temporal behavior. To do this, we used the results of the nonparametric one-sample Kolmogorov-Smirnov (K-S) test performed by Getman et al. (2005a). The results of the K-S test, $P_{\mathrm{K}-\mathrm{S}}$, strongly depend on the source count statistics and therefore on their count rate. For this reason, we compared the probabil- ities of variability $P_{\mathrm{var}}=\left(1-P_{\mathrm{K}-\mathrm{S}}\right)$ for the different classes of stars with count rates in definite ranges. The results are given in Table 13, where, for each class and for a given range of count rate (CR), we report the number of stars with $P_{\mathrm{var}}>99 \%$ with respect to the total number of stars of each class in the corresponding range of count rates. The percentage of variable stars is also indicated within brackets. Even if the statistics for each sample is quite poor, the percentages indicate that the different classes have very similar fractions of variable stars. In practice, when observed with high statistics, all the sources are variable, independently of their class.

\section{CLASS 0-I: COUNTERPARTS AT OTHER WAVELENGTHS}

We list in Table 14 the counterparts of the Class $0-$ I objects identified in this work that have been detected in X-rays (the COUP survey), JHKL bands (Lada et al. 2000; Muench et al. 2002, hereafter LMH00 and MLLA02, respectively), 10 and $20 \mu \mathrm{m}$ (Robberto et al. 2005a, hereafter RBP05), and in the $11.7 \mu \mathrm{m}$ (Smith et al. 2005, hereafter SBS05). None of our candidate Class 0-I sources are in the list of $1.3 \mathrm{~cm}$ radio sources of Zapata et al. (2004b).

The JHKL photometric survey from Lada et al. (2000) included 391 stars in the central $6.55^{\prime} \times 6.55^{\prime}$ of the Trapezium cluster. By selecting stars with $K-L>1.5$, they obtained a list of 78 candidate protostars. We correlated this list with our catalog using a matching radius of $1.2^{\prime \prime}$. As expected, the Lada et al. (2000) sample, selected on the basis of the $K-L$ color, is larger than our sample, for which the selection requires at least two colors computed using the $K$ and the four IRAC magnitudes. For this reason, we find that only 4 of the 78 Lada et al. (2000) candidate protostars are in common with our Class 0 -Ia stars and 9 with our Class $0-$ Ib stars. Among the remaining 65 objects, 16 cannot be Class $0-$ I objects since they have an optical counterpart in our catalog, and 27 have [3.6] $-[4.5]<0.7$ and are therefore consistent with star-disk systems (Class II) rather than protostars; for the remaining 22 Lada et al. (2000) candidate protostars, the available magnitudes do not satisfy our selection criteria.

Among our Class 0-I objects, only five (Class $0-\mathrm{Ib}$ ) have been detected at 10 and $20 \mu \mathrm{m}$ by Robberto et al. (2005a); of these five, two are in the list of the embedded objects previously studied in 

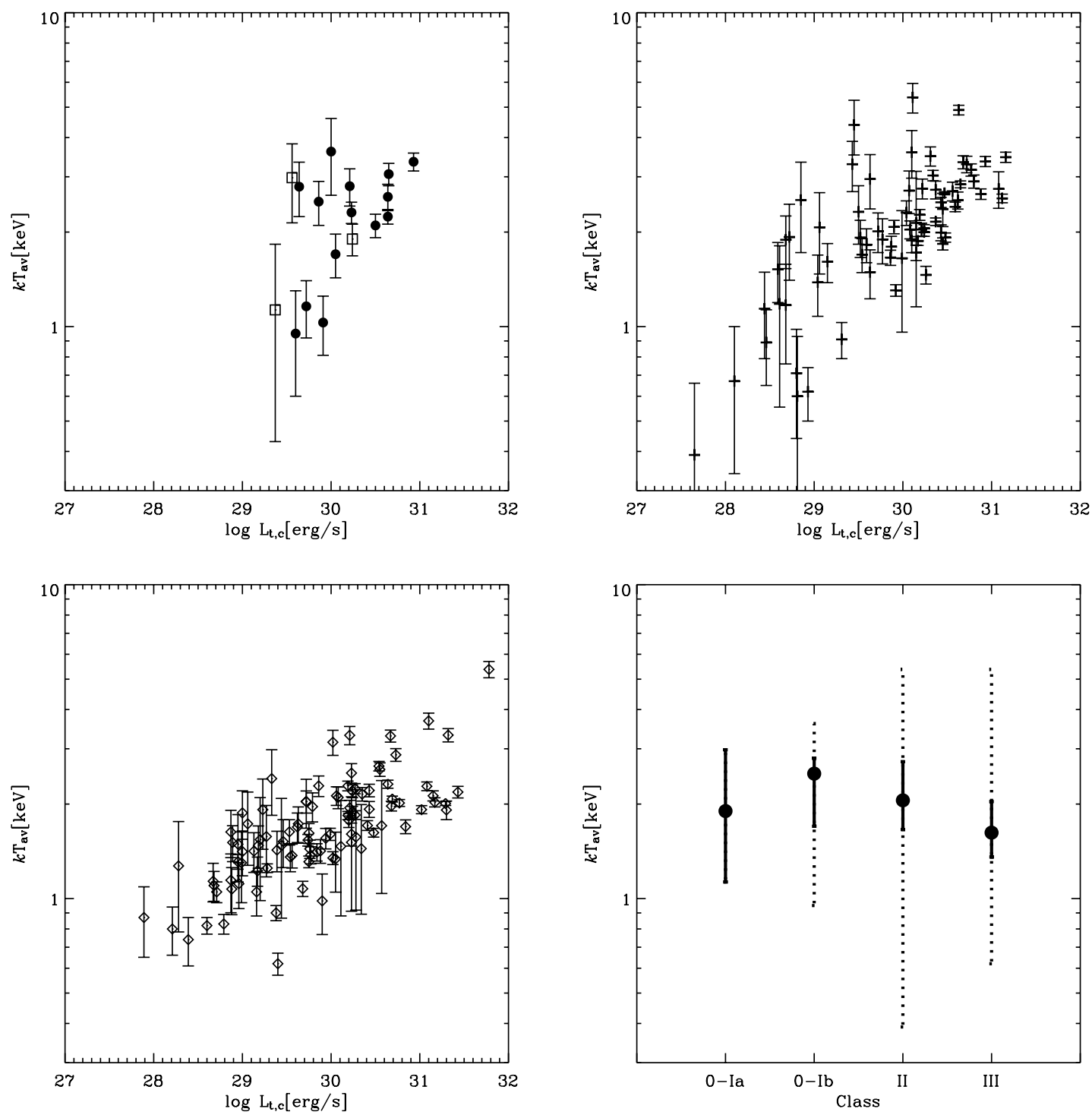

FIG. 13. - The $k T_{\mathrm{av}}$ values as a function of the unabsorbed X-ray luminosity $L_{t, c}$ for the X-ray-detected objects, plotted separately for Class $0-\mathrm{I}$, II, and III stars, in the first three panels. Symbols are as in Fig. 5 . The bottom right panel shows, for each class, the median values of the average plasma temperature distributions indicated by the big black filled circles. Solid bars indicate values of the temperature distributions between the first and third quartiles, while dashed lines (partially hidden by solid bars) indicate values between the minimum and the maximum of the distributions of the $k T_{\text {av }}$ values. Note that only objects with errors in $k T_{\text {av }}$ smaller than $1 \mathrm{keV}$ were considered.

Smith et al. (2004, hereafter SBS04) in the OMC-1S region at 8.8, 11.7 , and $18.8 \mu \mathrm{m}$.

We interpolated the flux conversion coefficients given in Lada et al. (2006) to 10 and $20 \mu \mathrm{m}$ and computed the corresponding fluxes. SEDs with all the available fluxes for these five objects are shown in Figure 14.

In order to understand the properties of our sample of Class $0-\mathrm{I}$ objects, we also considered the compact radio sources detected by Zapata et al. (2004b) and Zapata et al. (2004a) using, respectively, $1.3 \mathrm{~cm}$ (in a region of $30^{\prime \prime} \times 30^{\prime \prime}$ around OMC-1S) and

TABLE 13

X-Ray Variable Sources of Different Evolutionary Class Stars IN THE ONC WITH $P_{\mathrm{K}-\mathrm{S}}>99 \%$

\begin{tabular}{cclcc}
\hline \hline $\log (\mathrm{CR})$ & Class 0/Ia & \multicolumn{1}{c}{ Class 0/Ib } & \multicolumn{1}{c}{ Class II } & Class III \\
\hline$[-5,-4] \ldots \ldots \ldots \ldots$ & $2 / 4(50 \%)$ & $0 / 2(0 \%)$ & $1 / 4(25 \%)$ & $0 / 2(0 \%)$ \\
{$[-4,-3] \ldots \ldots \ldots \ldots$.} & $5 / 6(83 \%)$ & $6 / 9(66 \%)$ & $15 / 28(53 \%)$ & $19 / 30(63 \%)$ \\
{$[-3,-2] \ldots \ldots \ldots \ldots$} & $\ldots$ & $7 / 7(100 \%)$ & $38 / 38(100 \%)$ & $46 / 50(92 \%)$ \\
\hline
\end{tabular}

$3.6 \mathrm{~cm}$ observations (in a region of $4^{\prime} \times 4^{\prime}$ around the Trapezium cluster) performed with the Very Large Array. With a matching radius of $1^{\prime \prime}$, we retrieved in our catalog 7 of the 11 radio sources at $1.3 \mathrm{~cm}$ and 67 of the 77 compact $3.6 \mathrm{~cm}$ sources.

None of the Zapata et al. (2004a, 2004b) matched sources are in our list of Class $0-\mathrm{I}$ objects. The absence of $1 \mathrm{~cm}$ radio counterparts in our protostar sample might imply that the radio sources are mainly magnetically active nonthermal Class III stars rather than thermal protostellar outflows.

Note that the northeast region of the COUP FOV, where most of our candidate Class $0-\mathrm{I}$ objects are concentrated, is not included in the FOV of the literature sources mentioned in this section. For this reason, the SEDs of Class $0-$ I objects in this region cannot yet be extended to wavelengths longer than $8 \mu \mathrm{m}$, which is crucial to confirm (or refute) their protostellar nature.

Finally, our list of candidate Class 0 -I protostars includes only two objects (COUP sources 420 and 484) of the list of stars studied in Grosso et al. (2005), located at the bottom edge of the $\mathrm{OMC}-1 \mathrm{~S}$ region, i.e., far from the density peak and its associated nebular emission. We know that the absence of IRAC protostars 
TABLE 14

Literature Class 0/I Counterparts

\begin{tabular}{|c|c|c|c|c|c|c|c|c|}
\hline Sequence Number & Class & COUP & LMH00 & MLLA02 & SBS04 & SBS04 Name & SBS05 & RBP05 \\
\hline 1 & $0 /$ Ia & $\ldots$ & $\ldots$ & $\ldots$ & $\ldots$ & $\ldots$ & $\ldots$ & $\ldots$ \\
\hline 2 & $0 /$ Ia & $\ldots$ & $\ldots$ & $\ldots$ & $\ldots$ & $\ldots$ & $\ldots$ & $\ldots$ \\
\hline 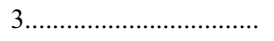 & $0 /$ Ia & 274 & $\ldots$ & $\ldots$ & $\ldots$ & $\ldots$ & $\ldots$ & $\ldots$ \\
\hline 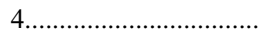 & $0 /$ Ia & $\ldots$ & $\ldots$ & $\ldots$ & $\ldots$ & $\ldots$ & $\ldots$ & $\ldots$ \\
\hline 5 & $0 /$ Ia & $\ldots$ & TPSC 63 & 771 & $\ldots$ & $\ldots$ & $\ldots$ & $\ldots$ \\
\hline 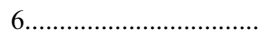 & $0 /$ Ia & $\ldots$ & $\ldots$ & $\ldots$ & $\ldots$ & $\ldots$ & $\ldots$ & $\ldots$ \\
\hline 7 & 0/Ia & 484 & TPSC 50 & $\ldots$ & $\ldots$ & $\ldots$ & $\ldots$ & $\ldots$ \\
\hline 8 & 0/Ia & $\ldots$ & $\ldots$ & $\ldots$ & $\ldots$ & $\ldots$ & $\ldots$ & $\ldots$ \\
\hline 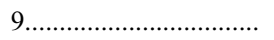 & 0/Ia & 696 & TPSC 59 & $\ldots$ & $\ldots$ & $\ldots$ & $\ldots$ & $\ldots$ \\
\hline $10 \ldots \ldots \ldots \ldots \ldots \ldots \ldots \ldots \ldots \ldots$ & $0 /$ Ia & 702 & $\ldots$ & $\ldots$ & $\ldots$ & $\ldots$ & $\ldots$ & $\ldots$ \\
\hline 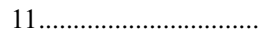 & $0 /$ Ia & 860 & $\ldots$ & $\ldots$ & $\ldots$ & $\ldots$ & $\ldots$ & $\ldots$ \\
\hline $12 \ldots \ldots \ldots$ & 0/Ia & 859 & $\ldots$ & $\ldots$ & $\ldots$ & $\ldots$ & $\ldots$ & $\ldots$ \\
\hline $13 \ldots \ldots \ldots \ldots \ldots \ldots \ldots \ldots \ldots$ & $0 /$ Ia & $\ldots$ & TPSC 56 & 950 & $\ldots$ & $\ldots$ & $\ldots$ & $\ldots$ \\
\hline 14 & $0 / \mathrm{Ia}$ & $\ldots$ & $\ldots$ & $\ldots$ & $\ldots$ & $\ldots$ & $\ldots$ & $\ldots$ \\
\hline 15 & $0 /$ Ia & $\ldots$ & $\ldots$ & $\ldots$ & $\ldots$ & $\ldots$ & $\ldots$ & $\ldots$ \\
\hline $16 \ldots$ & $0 / \mathrm{Ia}$ & 1048 & $\ldots$ & $\ldots$ & $\ldots$ & $\ldots$ & $\cdots$ & $\cdots$ \\
\hline 17. & $0 /$ Ia & 1115 & $\ldots$ & $\ldots$ & $\cdots$ & $\cdots$ & $\cdots$ & $\cdots$ \\
\hline 18 & $0 /$ Ia & $\ldots$ & $\ldots$ & $\ldots$ & $\ldots$ & $\ldots$ & $\ldots$ & $\ldots$ \\
\hline 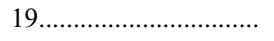 & $0 /$ Ia & 1197 & $\ldots$ & 988 & $\ldots$ & $\ldots$ & $\ldots$ & $\ldots$ \\
\hline 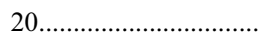 & 0/Ia & $\ldots$ & $\ldots$ & $\ldots$ & $\ldots$ & $\ldots$ & $\cdots$ & $\cdots$ \\
\hline $21 \ldots \ldots \ldots \ldots \ldots$ & $0 / \mathrm{Ia}$ & 1321 & $\ldots$ & 991 & $\ldots$ & $\ldots$ & $\cdots$ & $\cdots$ \\
\hline 22 & 0/Ia & $\ldots$ & $\ldots$ & $\ldots$ & $\ldots$ & $\ldots$ & $\ldots$ & $\ldots$ \\
\hline 23 & $0 /$ Ia & $\ldots$ & $\ldots$ & $\ldots$ & $\cdots$ & $\ldots$ & $\ldots$ & $\ldots$ \\
\hline 24 & $0 / \mathrm{Ib}$ & 103 & $\ldots$ & $\ldots$ & $\ldots$ & $\ldots$ & $\ldots$ & $\ldots$ \\
\hline 25 & $0 / \mathrm{Ib}$ & $\ldots$ & $\ldots$ & $\ldots$ & $\ldots$ & $\ldots$ & $\ldots$ & $\ldots$ \\
\hline 26 & $0 / \mathrm{Ib}$ & 167 & $\ldots$ & $\ldots$ & $\ldots$ & $\ldots$ & $\ldots$ & $\ldots$ \\
\hline 27 & $0 / \mathrm{Ib}$ & 209 & $\ldots$ & $\ldots$ & $\ldots$ & $\ldots$ & $\ldots$ & $\ldots$ \\
\hline 28 & $0 / \mathrm{Ib}$ & 407 & $\ldots$ & $\ldots$ & $\ldots$ & $\ldots$ & $\ldots$ & $\ldots$ \\
\hline $29 . \ldots \ldots \ldots$ & $0 / \mathrm{Ib}$ & 420 & TPSC 74 & $\ldots$ & $\ldots$ & $\ldots$ & $\ldots$ & MAX 18 \\
\hline $30 \ldots \ldots \ldots \ldots$ & $0 / \mathrm{Ib}$ & 448 & TPSC 65 & $\ldots$ & $\ldots$ & $\ldots$ & $\ldots$ & MAX 22 \\
\hline $31 \ldots \ldots$ & $0 / \mathrm{Ib}$ & 472 & TPSC 31 & $\ldots$ & $\ldots$ & $\ldots$ & $\ldots$ & $\ldots$ \\
\hline 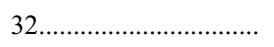 & $0 / \mathrm{Ib}$ & $\ldots$ & TPSC 3 & 369 & 10 & $134-330$ & 7 & MAX 40 \\
\hline 33 & $0 / \mathrm{Ib}$ & 548 & $\ldots$ & $\ldots$ & $\ldots$ & $\ldots$ & $\ldots$ & $\ldots$ \\
\hline $34 \ldots \ldots$ & $0 / \mathrm{Ib}$ & 570 & TPSC 35 & 797 & $\ldots$ & $\ldots$ & $\ldots$ & MAX 44 \\
\hline $35 \ldots \ldots \ldots \ldots \ldots \ldots \ldots \ldots$ & $0 / \mathrm{Ib}$ & $\ldots$ & TPSC 78 & $\ldots$ & 1 & $138-340$ & 16 & MAX 46 \\
\hline 36 & $0 / \mathrm{Ib}$ & 595 & $\ldots$ & $\ldots$ & $\ldots$ & $\ldots$ & $\ldots$ & $\ldots$ \\
\hline 37................................. & $0 / \mathrm{Ib}$ & $\ldots$ & $\ldots$ & $\ldots$ & $\ldots$ & $\ldots$ & $\ldots$ & $\ldots$ \\
\hline 38 & $0 / \mathrm{Ib}$ & 1030 & TPSC 41 & $\ldots$ & $\ldots$ & $\ldots$ & $\ldots$ & $\ldots$ \\
\hline 39. & $0 / \mathrm{Ib}$ & 1054 & $\ldots$ & $\ldots$ & $\ldots$ & $\ldots$ & $\ldots$ & $\ldots$ \\
\hline 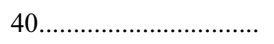 & $0 / \mathrm{Ib}$ & 1094 & TPSC 73 & 1001 & $\ldots$ & $\ldots$ & $\ldots$ & $\ldots$ \\
\hline 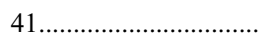 & $0 / \mathrm{Ib}$ & 1170 & TPSC 44 & 990 & $\ldots$ & $\ldots$ & $\ldots$ & $\ldots$ \\
\hline $42 \ldots \ldots \ldots$ & $0 / \mathrm{Ib}$ & 1188 & $\ldots$ & $\ldots$ & $\ldots$ & $\ldots$ & $\ldots$ & $\ldots$ \\
\hline $43 \ldots \ldots \ldots$ & $0 / \mathrm{Ib}$ & 1364 & $\ldots$ & $\ldots$ & $\cdots$ & $\ldots$ & $\ldots$ & $\cdots$ \\
\hline 44 & $0 / \mathrm{Ib}$ & 1480 & $\ldots$ & $\ldots$ & $\ldots$ & $\ldots$ & $\cdots$ & $\cdots$ \\
\hline 45 & $0 / \mathrm{Ib}$ & 1565 & $\ldots$ & $\ldots$ & $\ldots$ & $\ldots$ & $\ldots$ & $\ldots$ \\
\hline
\end{tabular}

near OMC-1S and in the BN/KL region is due to nebular contamination and crowding, not to the real absence of protostars in these two high-density regions. In addition, we cannot classify the COUP sources 582, 594, 615, 633, 641, 659, and 667, indicated in Grosso et al. (2005) as embedded YSOs in a subcluster, since they do not have any counterpart at the IRAC bands.

\section{SUMMARY AND CONCLUSIONS}

We have selected a sample of 45 candidate Class $0-$ I objects in the ONC field observed in the X-rays by COUP using new deep $J H K$, [3.6], [4.5], [5.8], and [8.0] photometry obtained at the ISPI at CTIO $4 \mathrm{~m}$ telescope and from Spitzer IRAC observations. New deep optical observations taken with the WFPC2 camera of HST and the WFI camera of ESO $2.2 \mathrm{~m}$ were also used to reject candidate Class $0-\mathrm{I}$ objects with optical counterparts.

We distinguish between candidate protostars with SEDs increasing monotonically from $K$ up to $8 \mu \mathrm{m}$, indicated as Class $0-\mathrm{Ia}$, and candidate protostars with SED increasing from $K$ up to $4.5 \mu \mathrm{m}$ and decreasing at longer wavelengths, indicated as Class $0-\mathrm{Ib}$, since the different slope of the SED at wavelengths larger than $4.5 \mu \mathrm{m}$ could be an indicator of a different density in the circumstellar material.

Out of the 23 Class 0 -Ia stars, 10 have been detected in the X-rays with COUP, while out of the 22 Class $0-I b$ stars, 18 have been detected; we are thus in the position to study, for the first time, the X-ray properties of a significant and homogeneous sample of candidate protostars.

Samples of bona fide more evolved Class II and III stars in the same region have also been selected in order to study the time and mass evolution of X-ray activity.

Our principal result is that Class 0-Ia stars are significantly less luminous in X-rays, in both the total and hard bands, than more evolved Class II stars with mass larger than $0.5 M_{\odot}$, while Class 0-Ib stars show X-ray luminosities similar to Class II stars; 

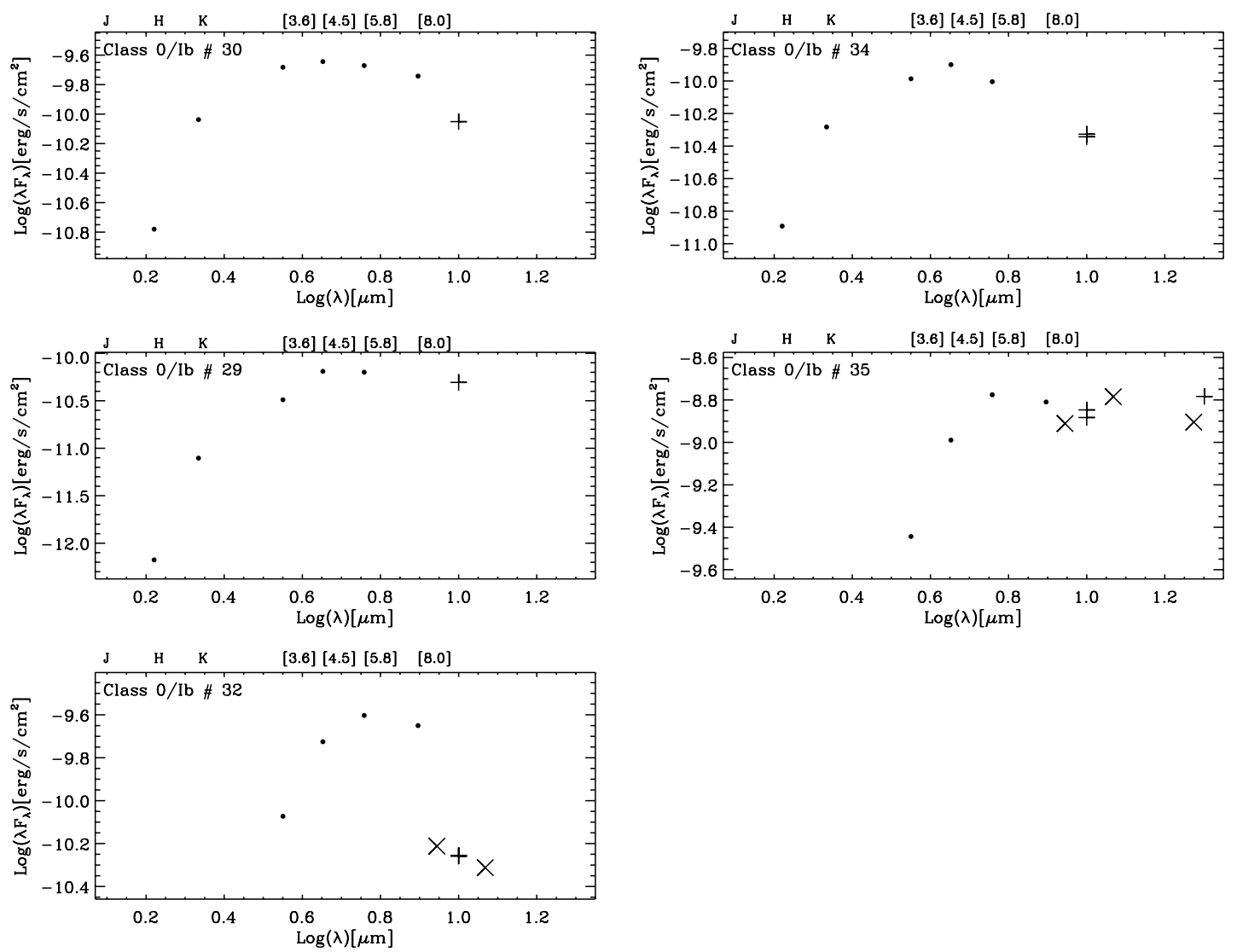

FIG. 14.-SED of Class 0-I candidate objects in the Robberto et al. (2005a) list with photometry at 10 and/or $20 \mu$ m. Filled circles indicate fluxes computed using the magnitudes from our catalog, plus signs correspond to the magnitudes at 10 and $20 \mu \mathrm{m}$ from the Robberto et al. (2005a) catalog, and crosses correspond to the magnitudes at 8.8,11.7, and $18.8 \mu \mathrm{m}$ from the Smith et al. (2004) catalog. Multiple values at $10 \mu \mathrm{m}$ are relative to the two different nights of observation in Robberto et al. (2005a).

finally, our data confirm previous determinations (Neuhauser 1997; Flaccomio et al. 2003c; Preibisch et al. 2005) that Class III stars with masses $>0.3 M_{\odot}$ are more X-ray luminous than Class II objects, even if the result is quite marginal with our data.

Our result that Class 0 -Ia objects are less luminous in X-rays than objects of other classes supports the hypothesis that the onset of X-ray emission occurs at a very early stage, when the objects show an increasing SED at least up to $8.0 \mu \mathrm{m}$. The lack of a detailed theory of protostellar evolution does not allow us to interpret the difference in the evolutionary stage between Class 0 -Ia and $0-\mathrm{Ib}$ protostars. Although Class $0-\mathrm{Ib}$ objects are selected as candidate protostars, our data suggest that this sample could be dominated by reddened Class II stars. If we consider the X-ray limiting sensitivity in Giardino et al. (2007), we deduce that our results are in agreement with the nondetection of Class 0 protostars in Serpens.

The X-ray spectral properties of Class 0 -Ia and $0-\mathrm{Ib}$ are similar to those of the more evolved Class II and III objects except for a larger absorption likely due to the enhanced local density surrounding protostellar objects. The different evolutionary classes also show similar X-ray variability characteristics, and the protostellar variability is indistinguishable from $\mathrm{T}$ Tauri variability. This result supports the XEST determination that variability of Class II and III stars is the same (Stelzer et al. 2007) and the COUP determination that $\mathrm{T}$ Tauri $\mathrm{M}$ stars have the same variability as more massive stars (Caramazza et al. 2007). Also related is the COUP result that YSOs with high X-ray luminosities have the same first ionization potential-related elemental abundances as older flaring stars (Maggio et al. 2007).

All of these results support the general theme that there is only one mechanism in YSO X-ray production at energies $\gtrsim 1 \mathrm{keV}$, and that this mechanism arises from stellar magnetic activity, which does not depend on accretion or disks. The various recent arguments (Kastner et al. 2002; Schmitt et al. 2005; Güdel et al. 2007; Argiroffi et al. 2007; Huenemoerder et al. 2007; Grosso et al. 2007) that accretion accounts for some X-ray emission and line ratios probably only refer to soft components that are not accessible to our study (Feigelson et al. 2007).

This work is based on observations made with Hubble Space Telescope and the Spitzer Space Telescope, which is operated by the Jet Propulsion Laboratory (JPL), California Institute of Technology under a contract with NASA. Partial support for this work was provided by ASI-INAF I/088/06/0 contract and by NASA through an award issued by JPL/Caltech. E. D. F. is supported by NASA contract NAS8-38252. This research has made use of NASA's Astrophysics Data System (ADS) Abstract Service and of the SIMBAD database, operated at CDS, Strasbourg, France. This research has also made use of data products from the Two Micron All Sky Survey (2MASS), which is a joint project of the University of Massachusetts and the Infrared Processing and Analysis Center, funded by the National Aeronautics and Space Administration and the National Science Foundation. These data were served by the NASA/IPAC Infrared Science Archive, which is operated by the Jet Propulsion Laboratory, California Institute of Technology, under contract with the National Aeronautics and Space Administration. We wish to thank Francesco Damiani and Konstantin Getman for helpful discussions. We also thank the anonymous referee for suggestions that helped improve this paper. 
Adams, F. C., Lada, C. J., \& Shu, F. H. 1987, ApJ, 312, 788

Allen, L., et al. 2007, in Protostars and Planets V, ed. B. Reipurth, D. Jewitt, \& K. Keil (Tucson: Univ. Arizona Press), 361

Allen, L. E., et al. 2004, ApJS, 154, 363

Andre, P., Ward-Thompson, D., \& Barsony, M. 1993, ApJ, 406, 122

Argiroffi, C., Maggio, A., \& Peres, G. 2007, A\&A, 465, L5

Bally, J., Stark, A. A., Wilson, R. W., \& Langer, W. D. 1987, ApJ, 312, L45

Caramazza, M., et al. 2007, A\&A, 471, 645

Cutri, R. M., et al. 2003, 2MASS All Sky Catalog of Point Sources

Damiani, F., Maggio, A., Micela, G., \& Sciortino, S. 1997a, ApJ, 483, 350 1997b, ApJ, 483, 370

D’Antona, F., \& Mazzitelli, I. 1994, ApJS, 90, 467

Favata, F., \& Micela, G. 2003, Space Sci. Rev., 108, 577

Fazio, G. G., et al. 2004, ApJS, 154, 10

Feigelson, E., Townsley, L., Güdel, M., \& Stassun, K. 2007, in Protostars and Planets V, ed. B. Reipurth, D. Jewitt, \& K. Keil (Tucson: Univ. Arizona Press), 313

Feigelson, E. D., \& Nelson, P. I. 1985, ApJ, 293, 192

Feigelson, E. D., et al. 2005, ApJS, 160, 379

Flaccomio, E., Micela, G., \& Sciortino, S. 2003a, A\&A, 397, 611

Flaccomio, E., et al. 2003b, ApJ, 582, 382 2003c, ApJ, 582, 398

Flaherty, K. M., Pipher, J. L., Megeath, S. T., Winston, E. M., Gutermuth, R. A., Muzerolle, J., Allen, L. E., \& Fazio, G. G. 2007, ApJ, 663, 1069

Getman, K. V., Feigelson, E. D., Garmire, G., Broos, P., \& Wang, J. 2007, ApJ, 654,316

Getman, K. V., et al. 2005a, ApJS, 160, 319 2005b, ApJS, 160, 353 2006, ApJS, 163, 306

Giardino, G., Favata, F., Micela, G., Sciortino, S., \& Winston, E. 2007, A\&A, 463,275

Grosso, N., Bouvier, J., Montmerle, T., Fernández, M., Grankin, K., \& Zapatero Osorio, M. R. 2007, A\&A, 475, 607

Grosso, N., et al. 2005, ApJS, 160, 530

Güdel, M., et al. 2007, A\&A, 468, 529

Gutermuth, R. A., et al. 2008, ApJ, 674, 336

Hamaguchi, K., et al. 2005, ApJ, 623, 291

Hartmann, L., et al. 2005, ApJ, 629, 881

Herbst, W., Bailer-Jones, C. A. L., Mundt, R., Meisenheimer, K., \& Wackermann, R. 2002, A\&A, 396, 513

Hillenbrand, L. A. 1997, AJ, 113, 1733 (H97)

Hillenbrand, L. A., Bauermeister, A., \& White, R. J. 2007, preprint (astro-ph/ 0703642)

Hillenbrand, L. A., \& Hartmann, L. W. 1998, ApJ, 492, 540

Huenemoerder, D. P., Kastner, J. H., Testa, P., Schulz, N. S., \& Weintraub, D. A. 2007, ApJ, 671, 592
REFERENCES

Kastner, J. H., Huenemoerder, D. P., Schulz, N. S., Canizares, C. R., \& Weintraub, D. A. 2002, ApJ, 567, 434

Kastner, J. H., et al. 2005, ApJS, 160, 511

Kenyon, S. J., Calvet, N., \& Hartmann, L. 1993, ApJ, 414, 676

Lada, C. J. 1987, in IAU Symp. 115, Star Forming Regions, ed. M. Peimbert \& J. Jugaku (Dordrecht: Reidel), 1

Lada, C. J., Muench, A. A., Lada, E. A., \& Alves, J. F. 2004, AJ, 128, 1254

Lada, C. J., \& Wilking, B. A. 1984, ApJ, 287, 610

Lada, C. J., et al. 2000, AJ, 120, 3162 (LMH00) 2006, AJ, 131, 1574

Maggio, A., et al. 2007, ApJ, 660, 1462

Megeath, S. T., et al. 2004, ApJS, 154, 367 2005, in IAU Symp. 227, Massive Star Birth: A Crossroads of Astrophysics, ed. R. Cesaroni et al. (Cambridge: Cambridge Univ. Press), 383

Muench, A. A., Lada, E. A., Lada, C. J., \& Alves, J. 2002, ApJ, 573, 366 (MLLA02)

Neuhauser, R. 1997, Science, 276, 1363

Ozawa, H., Grosso, N., \& Montmerle, T. 2005, A\&A, 429, 963

Preibisch, T., \& Feigelson, E. D. 2005, ApJS, 160, 390

Preibisch, T., et al. 2005, ApJS, 160, 401

Reach, W. T., et al. 2005, PASP, 117, 978

Rebull, L. M., et al. 2000, AJ, 119, 3026

Robberto, M., Beckwith, S. V. W., \& Panagia, N. 2002, ApJ, 578, 897

Robberto, M., et al. 2005a, AJ, 129, 1534 (RBP05) 2005b, BAAS, 37, 1404

Schmitt, J. H. M. M., Robrade, J., Ness, J.-U., Favata, F., \& Stelzer, B. 2005, A\&A, 432, L35

Schmitt, J. H. M. M., et al. 1990, ApJ, 365, 704

Smith, N., Bally, J., Shuping, R. Y., Morris, M., \& Hayward, T. L. 2004, ApJ, 610, L117 (SBS04)

Smith, N., Bally, J., Shuping, R. Y., Morris, M., \& Kassis, M. 2005, AJ, 130, 1763 (SBS05)

Stelzer, B., \& Schmitt, J. H. M. M. 2004, A\&A, 418, 687

Stelzer, B., et al. 2007, A\&A, 468, 463

Tsuboi, Y., et al. 2001, ApJ, 554, 734

Tsujimoto, M., Kobayashi, N., \& Tsuboi, Y. 2005, AJ, 130, 2212

Werner, M. W., et al. 2004, ApJS, 154, 1

Whitney, B. A., Wood, K., Bjorkman, J. E., \& Cohen, M. 2003, ApJ, 598, 1079 Wolk, S. J., et al. 2005, ApJS, 160, 423

Zapata, L. A., Rodríguez, L. F., Kurtz, S. E., \& O’Dell, C. R. 2004a, AJ, 127, 2252

Zapata, L. A., Rodríguez, L. F., Kurtz, S. E., O’Dell, C. R., \& Ho, P. T. P. 2004b, ApJ, 610, L121 